\title{
Stromal cell-derived DEL-1 inhibits Tfh cell activation and inflammatory arthritis
}

\author{
Hui Wang, ${ }^{1}$ Xiaofei Li, ${ }^{1}$ Tetsuhiro Kajikawa, ${ }^{1}$ Jieun Shin, ${ }^{1}$ Jong-Hyung Lim, ${ }^{1}$ Ioannis Kourtzelis, ${ }^{2,3}$ Kosuke Nagai, ${ }^{2}$ Jonathan M. Korostoff, \\ Sylvia Grossklaus, ${ }^{2}$ Ronald Naumann, ${ }^{5}$ Triantafyllos Chavakis, ${ }^{2,6}$ and George Hajishengallis ${ }^{1}$ \\ 'Department of Basic and Translational Sciences, Penn Dental Medicine, University of Pennsylvania, Philadelphia, Pennsylvania, USA. Institute for Clinical Chemistry and Laboratory Medicine, Faculty of \\ Medicine, Technische Universität Dresden, Dresden, Germany. ${ }^{3}$ Hull York Medical School, York Biomedical Research Institute, University of York, York, United Kingdom. ${ }^{4}$ Department of Periodontics, Penn \\ Dental Medicine, University of Pennsylvania, Philadelphia, Pennsylvania, USA. ${ }^{5}$ Transgenic Core Facility, Max Planck Institute for Molecular Cell Biology and Cenetics, Dresden, Germany. ${ }^{6}$ Centre \\ for Cardiovascular Science, Queen's Medical Research Institute, University of Edinburgh, Edinburgh, United Kingdom.
}

\begin{abstract}
The secreted protein developmental endothelial locus 1 (DEL-1) regulates inflammatory cell recruitment and protects against inflammatory pathologies in animal models. Here, we investigated DEL-1 in inflammatory arthritis using collageninduced arthritis (CIA) and collagen Ab-induced arthritis (CAIA) models. In both models, mice with endothelium-specific overexpression of DEL-1 were protected from arthritis relative to WT controls, whereas arthritis was exacerbated in DEL-1deficient mice. Compared with WT controls, mice with collagen VI promoter-driven overexpression of DEL-1 in mesenchymal cells were protected against CIA but not CAIA, suggesting a role for DEL-1 in the induction of the arthritogenic Ab response. Indeed, DEL-1 was expressed in perivascular stromal cells of the lymph nodes and inhibited Tfh and germinal center B cell responses. Mechanistically, DEL-1 inhibited DC-dependent induction of Tfh cells by targeting the LFA-1 integrin on T cells. Overall, DEL-1 restrained arthritis through a dual mechanism, one acting locally in the joints and associated with the antirecruitment function of endothelial cell-derived DEL-1; the other mechanism acting systemically in the lymph nodes and associated with the ability of stromal cell-derived DEL-1 to restrain Tfh responses. DEL-1 may therefore be a promising therapeutic for the treatment of inflammatory arthritis.
\end{abstract}

\section{Introduction}

Inflammatory arthritides, such as rheumatoid arthritis (RA), are chronic inflammatory diseases that progressively affect the synovial joints in susceptible individuals. RA is characterized by production of autoantibodies against extracellular matrix proteins and other targets, synovial inflammation and hyperplasia, and progressive cartilage and bone erosion (1). Remission of RA symptoms is potentially attainable with antiinflammatory drugs, including biologic agents (1). Further advances in understanding regulatory mechanisms in RA pathogenesis may lead to new therapeutics with improved outcomes.

In RA, the induction of autoantibody generation in lymph nodes (LNs) draining target joints involves presentation of self-peptides (e.g., derived from citrullinated matrix proteins) by antigen-presenting cells (APCs) to Tfh cells. The latter then home to $B$ cell follicles to stimulate B cells to produce Abs against selfproteins (2). Tfh cells are characterized by the defining transcription factor B cell lymphoma 6 (BCL6) and by high-level expression of CXCR5, which promotes their homing to B cell follicles, as well as by expression of programmed cell death 1 (PD1), ICOS, and IL-21 $(3,4)$. These features of Tfh cells promote their ability

Authorship note: TC and $\mathrm{CH}$ are co-senior authors and contributed equally to this work. Conflict of interest: The authors have declared that no conflict of interest exists. Copyright: (c) 2021, American Society for Clinical Investigation.

Submitted: April 15, 2021; Accepted: August 12, 2021; Published: October 1, 2021. Reference information: / Clin Invest. 2021;131(19):e150578.

https://doi.org/10.1172/JCl150578. to interact with and provide help to B cells in the germinal centers (GCs) of the B cell follicles (5).

$\mathrm{Tfh}$ cell differentiation from naive $\mathrm{CD} 4^{+} \mathrm{T}$ cells in the $\mathrm{T}$ cell zone of LNs requires strong $\mathrm{T}$ cell receptor (TCR) signals derived from antigens presented by DCs in the context of MHC class II and/or sustained interactions with DCs, thereby prolonging costimulatory molecule engagement (e.g., ICOS-ICOS ligand interaction) or the exposure to critical cytokines (e.g., IL-6, IL-21; refs. 4,6$)$. High binding strength of the TCR to its cognate ligand (MHC class II-bound peptide) is selectively associated with Tfh differentiation over other effector $\mathrm{T}$ cell fates (6).

The $\beta 2$ integrin leukocyte function-associated antigen-1 (LFA1 ; CD11a/CD18) is required for optimal CD $4^{+} \mathrm{T}$ cell priming in LNs (7). Additionally, the binding of LFA-1 on T cells to ICAMs on APCs promotes sustained cognate T cell-APC interactions and lowers the threshold for antigen-specific T cell activation (5). Recently, LFA-1 was shown to promote the development and maintenance of Tfh cells (8). Specifically, in the context of TCR activation, LFA- 1 fosters the expression of BCL6, which is critical for early (prior to GC formation) DC-dependent Tfh cell differentiation and for the survival of Tfh cells within the GC niche (8). BCL6, moreover, represses the induction of differentiation of alternative $\mathrm{CD} 4^{+}$effector cells $(3,9)$.

RA-associated autoantibodies may bind to target antigens and form immune complexes in the joint, thereby contributing to the initiation of synovitis typified by increased recruitment of inflammatory cells (10). Leukocyte accumulation in the synovial compartment is primarily due to recruitment from the circulation 
rather than local proliferation (11). Recruited neutrophils and macrophages further contribute to synovitis by releasing matrixdegrading proteases and proinflammatory mediators (11-13). Synovium-infiltrating leukocytes, including $\mathrm{CD} 4^{+}$Th17 cells, activate resident fibroblasts, which in turn further propagate inflammation and local tissue destruction (11).

Developmental endothelial locus 1 (DEL-1) is a $52 \mathrm{kDa}$ protein comprising $3 \mathrm{~N}$-terminal EGF-like repeats and $2 \mathrm{C}$-terminal discoidin I-like domains; it is secreted by tissue-resident cells (e.g., mesenchymal stromal cells, endothelial cells, and certain macrophage subsets) and regulates both the initiation and resolution of inflammation (14). Specifically, DEL-1 binds LFA-1 ( $\alpha$ L $\beta 2$ integrin) and Mac-1 ( $\alpha \mathrm{M} \beta 2$ integrin) and antagonizes $\beta 2$ integrin-dependent leukocyte adhesion to endothelial ICAM-1, thereby restraining leukocyte recruitment (15-17). Consequently, genetic deficiency of DEL-1 in mice causes increased leukocyte infiltration in different inflammatory disease models $(15,16,18-21)$. DEL-1 also binds the $\alpha v \beta 3$ integrin by virtue of an RGD (Arg-Gly-Asp) site in its second EGF-like repeat (22) as well as the "eat-me" signal phosphatidylserine on apoptotic cells by means of its discoidin I-like domains $(21,23)$. These interactions bridge apoptotic cells to macrophages and promote apoptotic cell phagocytosis (efferocytosis) and inflammation resolution (21). Consistent with these homeostatic functions, DEL-1 protects against inflammatory disorders in preclinical mouse or nonhuman primate models, including inflammatory bone loss in periodontitis, experimental autoimmune encephalomyelitis, pulmonary inflammation and fibrosis, and allergic asthma (16, 18, 20, 24-26).

Given the antiinflammatory actions of DEL-1, we explored whether DEL-1 can also protect against inflammatory arthritis. By using 2 models of experimental arthritis (collagen-induced arthritis [CIA] and collagen Ab-induced arthritis [CAIA]) and DEL-1 transgenic and KO mice, we showed here that DEL-1 also restrained arthritis. The underlying protective mechanism involved not only the established capacity of DEL-1 to limit local inflammatory cell recruitment (to the joints) but, surprisingly, also its systemic effect to downregulate the induction of the arthritogenic $\mathrm{Ab}$ response in the LNs. LN stromal cell-derived DEL-1 inhibited DC-dependent Tfh induction by targeting the LFA- 1 integrin on T cells, thereby restraining $\mathrm{B}$ cell responses in GCs and the production of arthritogenic Abs that drove arthritis in the CIA model. The capacity of DEL-1 to interfere with arthritogenic mechanisms at local and systemic levels suggests that this molecule may be a promising therapeutic for the treatment of RA.

\section{Results}

DEL-1 deficiency exacerbates arthritis and inflammatory cell infiltration. To determine the role of DEL-1 in arthritis, we compared Del1-KO mice and WT littermate controls in 2 distinct experimental arthritis settings, the CIA and CAIA models. Del1-KO mice subjected to CIA developed more severe arthritis than their WT controls, as evidenced by significantly increased joint thickness (Figure 1A) and clinical score (Figure 1B). Del1-KO mice also displayed significantly elevated cellularity in the synovium (Figure $1 \mathrm{C}$ ) compared with WT littermate controls on day 42 . Histological analysis of the joints revealed that Del1-KO mice displayed loss of joint architecture with increased inflammatory cell infiltrate and synovial hyperplasia (Figure 1D) and increased loss of safranin-O-stained cartilage and roughening of the articular surface (Figure 1E) compared with WT controls. Because endothelial cell-derived DEL-1 antagonizes $\beta 2$ integrin-dependent leukocyte recruitment $(15-17,21)$, we examined whether the increased severity of arthritis in Del1-KO mice was associated with increased infiltration of neutrophils and macrophages. FACS analysis of cells harvested from the knee joints of Del1-KO and WT mice (Supplemental Figure 1A; supplemental material available online with this article; https://doi.org/10.1172/JCI150578DS1) revealed that Del1-KO mice indeed harbored significantly elevated numbers of neutrophils and macrophages (Figure $1 \mathrm{~F}$ ). Similar observations were made using the CAIA model; Del1-KO mice developed more severe arthritis clinically (Figure $1, \mathrm{G}$ and $\mathrm{H}$ ) and histologically (Figure 1, J and K), as well as increased synovial cellularity and infiltration with myeloid cells (Figure 1, I and L), relative to WT littermates. Therefore, endogenous DEL-1 attenuated joint inflammation and damage in 2 distinct mouse arthritis models.

Endothelium-specific overexpression of DEL-1 protects against CIA and CAIA. The findings of increased neutrophil and macrophage infiltration in the joints of Del1-KO mice relative to their WT littermates in both CIA and CAIA are consistent with the antirecruitment role of endothelial cell-derived DEL-1 (14-16, 21). To rigorously test the notion that endothelial cell-derived DEL-1 provides protection against arthritis, we engaged mice with endothelial cell-specific overexpression of DEL-1 (EC-Del1 mice; refs. 19, 27, 28). To better distinguish potential differences in arthritis susceptibility between EC-Del1 mice and WT littermate controls, we used a more severe CIA protocol (than used to compare Del1-KO and WT littermate mice) by increasing the dose of the CFA from 1 to $2 \mathrm{mg} /$ $\mathrm{mL}$ (details in Methods). CIA in EC-Del1 mice had reduced severity compared with WT littermates, as evidenced by significantly decreased joint thickness (Figure 2A) and clinical score (Figure 2B). EC-Del1 mice also displayed significantly decreased cellularity in the synovium compared with WT littermates on day 42 (Figure 2C). Histological analysis of the joints showed that EC-Del1 mice had decreased inflammatory cell infiltrate and synovial hyperplasia (Figure 2D) and cartilage degradation (Figure 2E) relative to WT littermates. FACS analysis of cells harvested from the knee joints indicated significantly decreased numbers of neutrophils and macrophages in the EC-Del1 group compared with the WT group (Figure $2 \mathrm{~F})$. Likewise, when EC-Del1 mice and WT littermate controls were subjected to CAIA, EC-Del1 mice displayed significantly decreased joint thickness (Figure 2G) and clinical score (Figure 2H) during the progression of arthritis, although not significantly after day 8, which signified the onset of the resolution phase of CAIA. Moreover, compared with WT controls, EC-Del1 mice displayed significantly decreased cellularity in the synovium (Figure 2I), reduced inflammatory cell infiltrate, synovial hyperplasia (Figure 2J), and cartilage degradation (Figure 2K). EC-Del1 mice also had significantly reduced neutrophil and macrophage accumulation on day 7 (Figure 2L). Taken together, mice with endothelial cell-specific overexpression of DEL-1 displayed reduced infiltration of neutrophils and macrophages in the joints associated with decreased severity of arthritis in both the CIA and CAIA models.

ColVI-Del1 mice are protected from CIA but not from CAIA. Despite the documented protection against arthritis conferred by endogenous DEL-1 (Figure 1), we could not exclude the involvement of additional mechanisms over and above those mediated by 
A

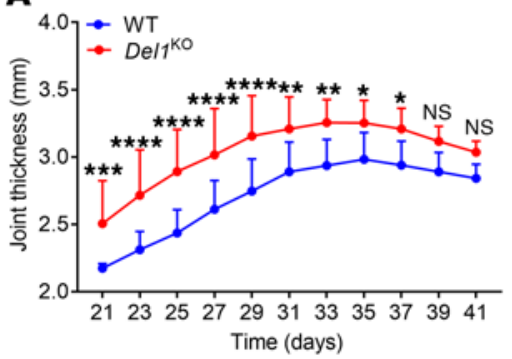

D

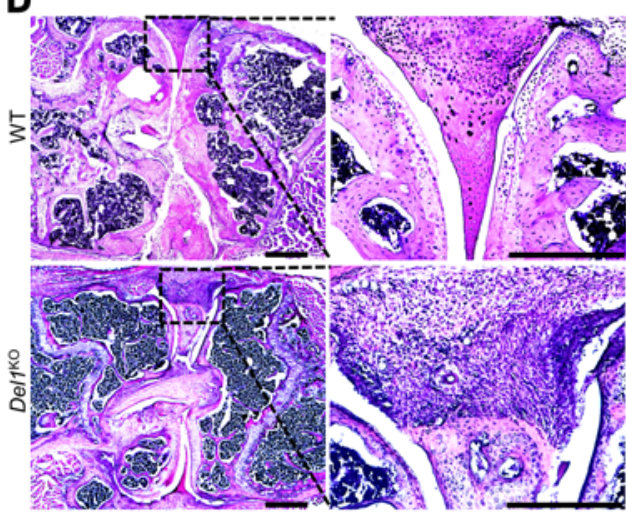

B

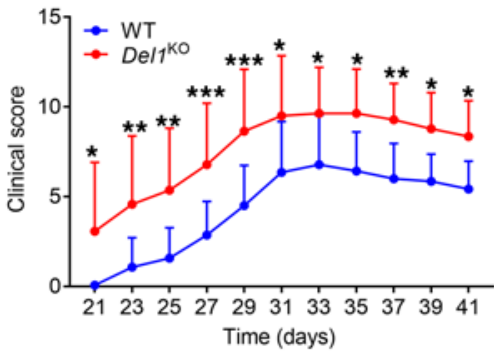

E

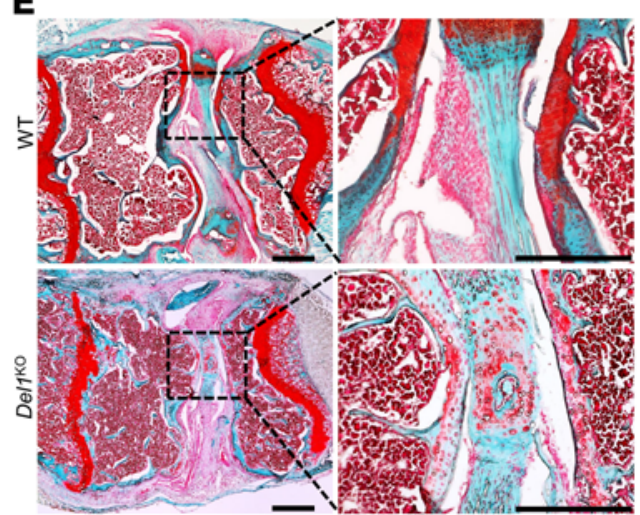

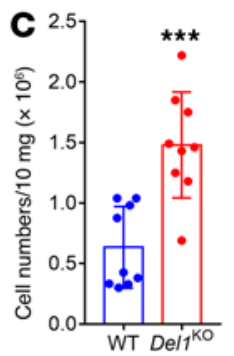

$\mathbf{F}$

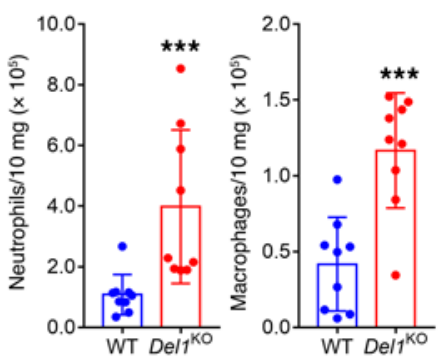

G

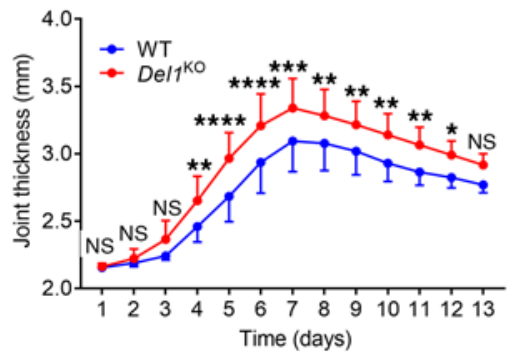

J

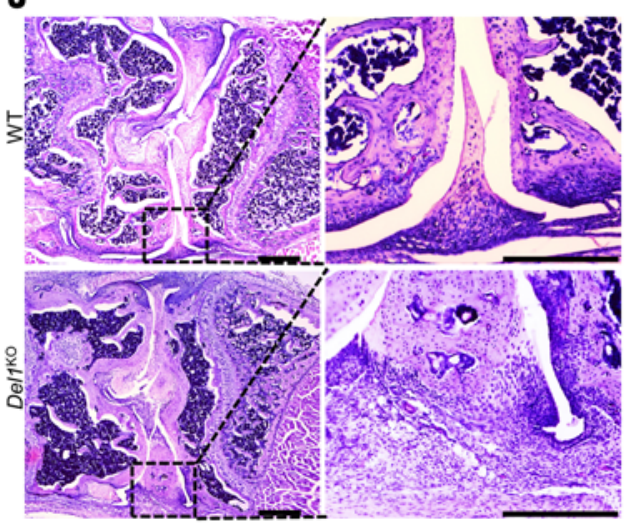

H

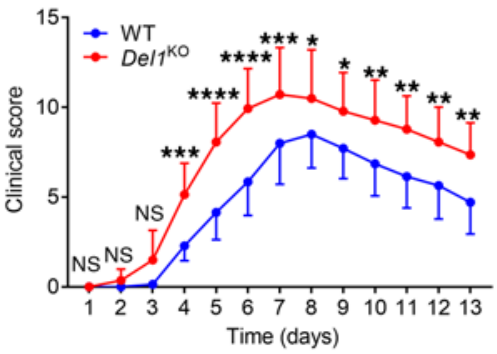

$\mathbf{K}$

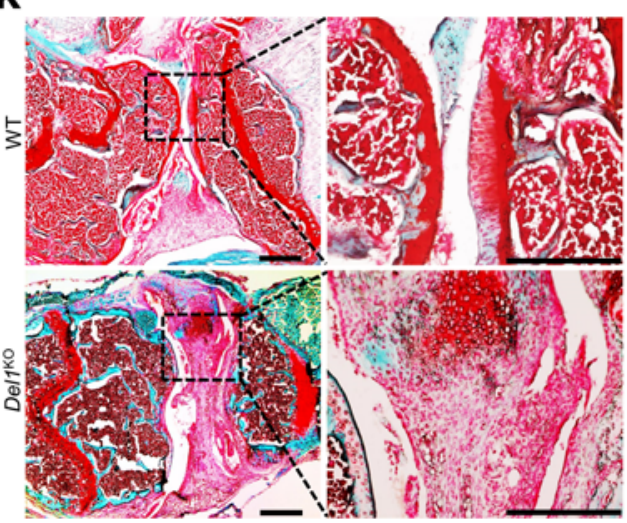

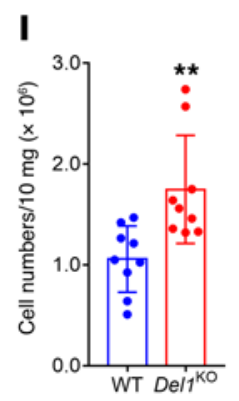

$\mathbf{L}$

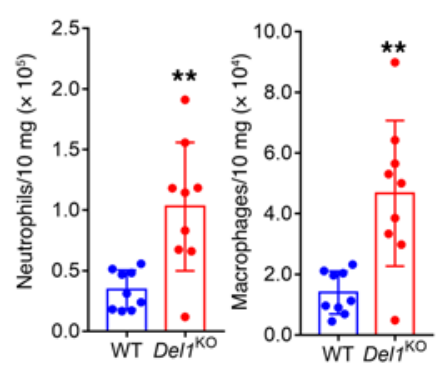

Figure 1. Del1-KO mice are more susceptible to CIA and CAIA relative to WT littermate controls. CIA was induced by i.d. injection of $2 \mathrm{mg} / \mathrm{mL}$ CII emulsified with 1 $\mathrm{mg} / \mathrm{mL}$ CFA into the tail of Del1-KO mice and WT littermates. Joint thickness (A) and clinical arthritis scores (B) were recorded at indicated time points. (C) Total cell numbers in the synovium of knee joints in Del7-KO and WT mice on day 42. H\&E (D) and safranin-O (E) staining of tissue sections from knee joints harvested on day 42. Scale bars: $500 \mu \mathrm{m}$. (F) Cell suspensions prepared from the synovium of knee joints (harvested on day 42) were processed for FACS to identify and quantify

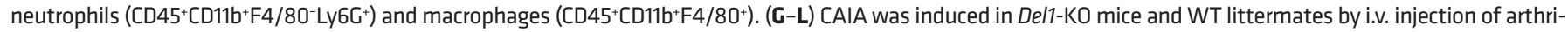
togenic mAbs ( $0.5 \mathrm{mg} /$ mouse) at day 0 , followed by i.p. injection of $50 \mu \mathrm{g}$ LPS on day 3. Joint thickness (C) and clinical arthritis scores (H) were recorded every day. (I) Total cell numbers in the synovium of knee joints in Del1-KO and WT mice on day 7. H\&E (J) and safranin-O (K) staining of tissue sections from knee joints harvested on day 7. Scale bars: $500 \mu \mathrm{m}$. (L) Cell suspensions prepared from the synovium of knee joints (harvested on day 7) were processed for FACS to identify and quantify

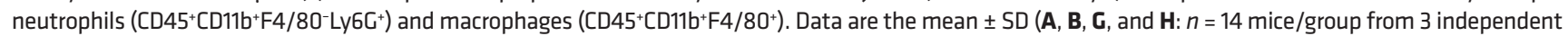
experiments; $\mathbf{C}, \mathbf{F}, \mathbf{I}$, and $\mathbf{L}: \mathbf{n}=9$ mice/group from 2 independent experiments). ${ }^{*} P<0.05 ;{ }^{* *} P<0.01 ;{ }^{* * *} P<0.001 ;{ }^{* * *} P<0.0001$. Two-way ANOVA with repeatedmeasures and Sidak's post tests for comparison with WT mice (A, B, G, and $\mathbf{H})$; Mann-Whitney $U$ test (C, F [left]); and Student's unpaired $t$ test (F [right], I, and L). 
endothelial DEL-1 (Figure 2). Since synovial fibroblasts also play an important role in inflammatory arthritis, including RA $(11,29)$, we assessed the contribution of mesenchymal/fibroblast-derived DEL1. To this end, using a collagen VI (ColVI) alpha1 promoter construct (30), we generated transgenic mice (ColVI-Del1 mice) overexpressing DEL-1 in mesenchymal cells, including synovial fibroblasts. Indeed, synovial fibroblasts isolated from the joints of ColVI-Del1 mice expressed significantly higher levels of DEL-1 mRNA and protein than those from WT littermates (Supplemental Figure 2).

ColVI-Del1 mice subjected to CIA (using the more severe protocol involving $2 \mathrm{mg} / \mathrm{mL}$ CFA) were protected from arthritis relative to WT littermate controls, as shown by significantly decreased joint thickness (Figure 3A) and clinical score (Figure 3B) during almost the entire recording period (days 22-41). ColVI-Del1 mice also exhibited significantly decreased cellularity in the synovium (Figure 3C) compared with WT littermates on day 42. Consistently, histological analysis of the joints revealed that ColVI-Del1 mice exhibited decreased inflammatory cell infiltrate and synovial hyperplasia (Figure 3D) and diminished cartilage destruction (Figure $3 \mathrm{E}$ ) relative to $\mathrm{WT}$ littermates.

If the DEL-1-mediated protective function accounting for these findings operates locally in the joints, ColVI-Del1 mice would be expected to also be protected from CAIA, where passive administration of premade arthritogenic Abs serves as a model for immune complex-mediated pathogenic effector responses in the joints (31). Strikingly, however, no differences were observed between ColVIDel1 mice and WT littermates in terms of CAIA severity as assessed by joint thickness and clinical score (Figure 3, G and $\mathrm{H}$ ). This finding suggested that the protective mechanism associated with the overexpression of DEL-1 from a ColVI alpha1 promoter might not operate effectively after arthritogenic Abs have been induced or administered passively. Given that the CAIA model bypassed the initial $\mathrm{T}$ cell priming by APCs and the ensuing $\mathrm{T}-\mathrm{B}$ cell interactions required for induction of anti-type II collagen (CII) Ab responses in the CIA model, we investigated whether DEL-1 can modulate the induction of the anti-CII Ab response in the latter model. To this end, we first analyzed the sera of ColVI-Del1 mice and their WT controls, which were subjected to CIA. ColVI-Del1 mice exhibited significantly lower levels of serum IgG1 and IgG2a (but not IgG2b) isotype-specific anti-CII Abs at all time points tested after day 7, as well as lower levels of CII-specific total IgG Abs on days 14 and 42 (Figure $3 \mathrm{~F}$ ). These data suggested that DEL-1 may modulate the arthritogenic $\mathrm{Ab}$ production.

ColvI-Del1 mice display reduced numbers of Tfh cells and GC-B cells in inguinal LNs upon CIA. After intradermal (i.d.) immunization with CII and CFA in the CIA model, the draining inguinal LNs react against the immunizing antigen with $\mathrm{T}$ cell activation and GC formation (32). We hypothesized that DEL-1 might regulate Ab production by modulating the numbers or function of Th cells in the inguinal LNs. To examine this, we performed FACS analysis of mononuclear cells from inguinal LNs harvested from ColVI-Del1 mice subjected to CIA or WT controls on day 10 after CIA. The analysis revealed that ColVI-Del1 mice had reduced frequencies and absolute numbers of Tfh cells $\left(\mathrm{CD} 4^{+} \mathrm{CD} 19^{-} \mathrm{CXCR} 5^{+} \mathrm{PD}-\mathrm{1}^{\text {hi }} \mathrm{BCL}-\right.$ $6^{\text {hi) }}$ as well as decreased expression of BCL6 per cell (MFI) relative to WT controls (Figure 4, A and B). Consistent with the essential role of Tfh cells in the generation of GCs (5), ColVI-Del1 mice also showed significantly decreased frequencies and numbers of GC-B cells $\left(\mathrm{CD} 4{ }^{-} \mathrm{CD} 19^{+} \mathrm{PNA}^{+} \mathrm{FAS}^{+}\right.$) in inguinal LNs (Figure $4, \mathrm{C}$ and $\mathrm{D}$ ), in line with the reduced capacity of these mice to elicit CII-specific IgG Abs (Figure 3F). Given that Th1 and Th17 cells are also important in the pathogenesis of arthritis (11), we examined whether these $\mathrm{T}$ cell effector subsets are differentially affected in ColVI-Del1 mice subjected to CIA relative to WT controls. However, the frequencies of Th1 and Th17 cells were similar in ColVI-Del1 and WT on day 10 after induction of CIA (Supplemental Figure 3). In steady-state inguinal LNs, 8-week-old ColVI-Del1 mice and WT controls exhibited comparable frequencies and absolute numbers of Th cells as well as BCL6 expression (MFI) (Figure 4, E and F). Therefore, DEL-1 can inhibit Tfh induction preferentially upon immunological stress, such as in the context of CIA.

DEL-1 inhibits DC-dependent Tfh cell differentiation by targeting the LFA-1 integrin on $T$ cells. We next investigated possible mechanism(s) whereby DEL-1 may affect Tfh cells. We first determined whether DEL-1 can inhibit induction of Tfh differentiation markers in a standard assay based on polyclonal stimulation of naive $\mathrm{CD} 4^{+} \mathrm{T}$ cells with anti-CD3/anti-CD28 and appropriate cytokine conditions (IL-6, anti-TGF- $\beta$, anti-IL-4, and anti-IFN- $\gamma$ ). In comparison with the Fc control, exogenously added DEL-1-Fc failed to inhibit the mRNA expression of the transcription factor BCL6, which is both necessary and sufficient for Tfh differentiation, and other important Tfh markers (IL-21 and CXCR5; refs. 3, 4, 9 and Supplemental Figure 4A). Moreover, DEL-1-Fc failed to influence the mRNA expression of B lymphocyte-induced maturation protein 1 (BLIMP-1; Supplemental Figure 4A), a transcription factor that inhibits Tfh differentiation (33). Consistently, DEL-1-Fc did not influence the ability of $\mathrm{CD} 4^{+} \mathrm{T}$ cells cultured under $\mathrm{Tfh}$ differentiation conditions to produce IL-21 protein (Supplemental Figure 4B). DEL-1 may thus not influence Tfh cell differentiation by direct action on $\mathrm{CD}^{+}$ T cells, which prompted us to consider whether DEL-1 can affect the priming interactions between DCs and T cells leading to Tfh cell differentiation. To address this possibility, we first examined whether DEL-1 has direct effects on DCs. Using LPS-activated bone marrow-derived DCs (BMDCs), we found that DEL-1-Fc failed to inhibit the expression of costimulatory molecules, including the ICOS ligand (Supplemental Figure 5A), or the production of IL-6 (Supplemental Figure 5B), factors involved in the ability of DCs to initiate Tfh cell differentiation $(4,34)$.

We then investigated whether DEL- 1 can affect DC-T cell interactions using a coculture system originally used for human cells (35, 36). Briefly, LPS-pretreated and washed BMDCs were cocultured for 7 days with naive $\mathrm{CD} 4^{+} \mathrm{T}$ cells stimulated with anti-CD3/CD28 in the presence or absence of DEL-1-Fc or Fc control. The generation of Tfh cells (defined as $\mathrm{CD} 4^{+} \mathrm{CXCR} 5^{+} \mathrm{PD}-1^{\text {hi }} \mathrm{BCL} 6^{\text {hi) }}$ was significantly higher when $\mathrm{CD} 4^{+} \mathrm{T}$ cells were cocultured with LPS-preactivated BMDCs than with non-preactivated BMDCs (Figure 5, A and B). Importantly, DEL-1-Fc but not Fc control caused a significant decrease in the percentage of generated Tfh cells in the LPS-preactivated BMDC-T cell coculture system (Figure 5, A and B). Interestingly, DEL-1[RGE]-Fc, a DEL-1 variant that does not interact with $\alpha v \beta 3$ integrin, caused a reduction in Tfh cell numbers similar to that observed with DEL-1-Fc (Figure 5, A and B).

The ability of DEL-1[RGE]-Fc to inhibit Tfh differentiation in the coculture system ruled out the $\alpha \mathrm{v} \beta 3$ integrin as a potential target of 
A

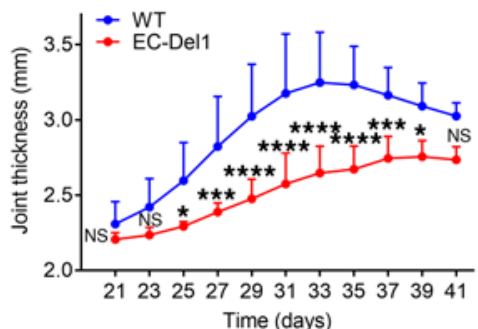

D

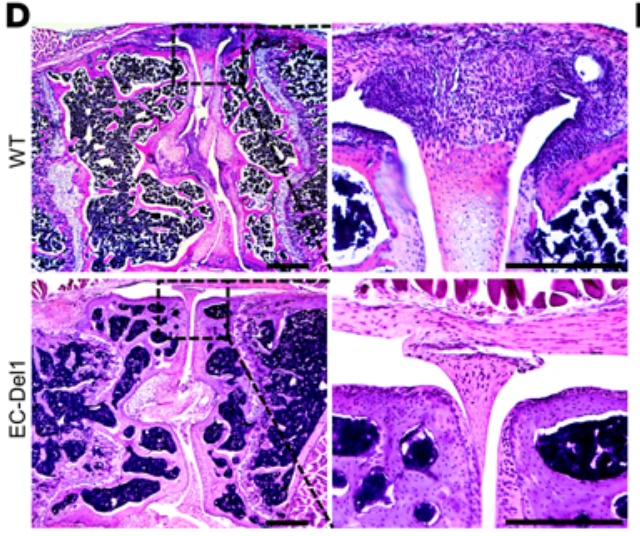

B

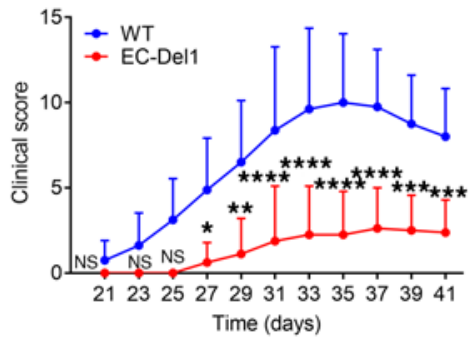

E

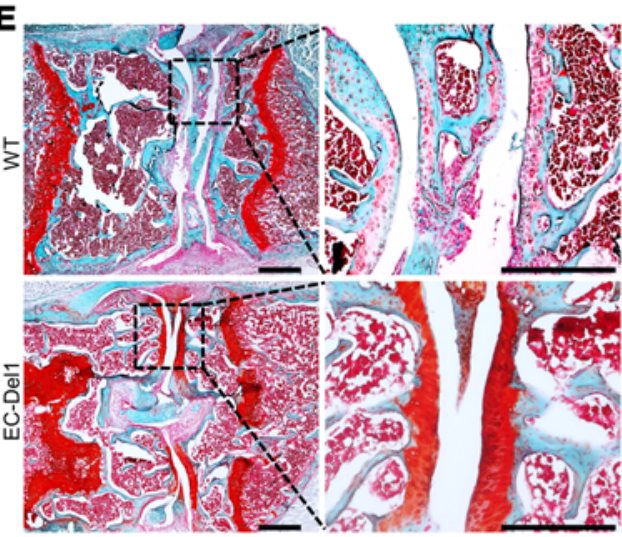

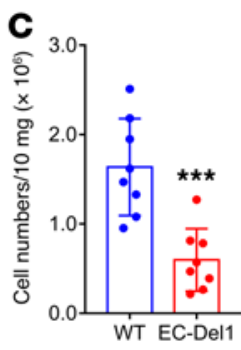

$\mathbf{F}$

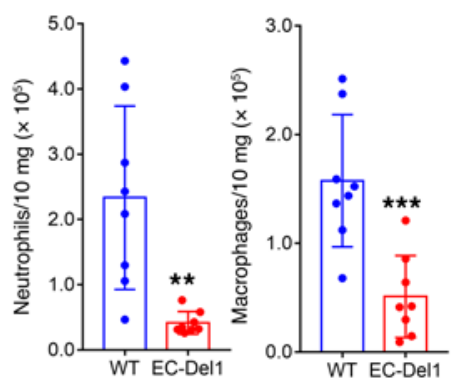

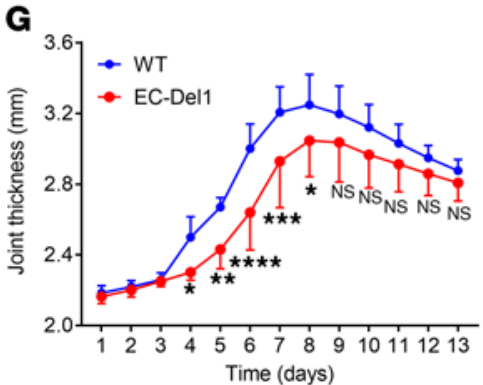

$\mathbf{J}$

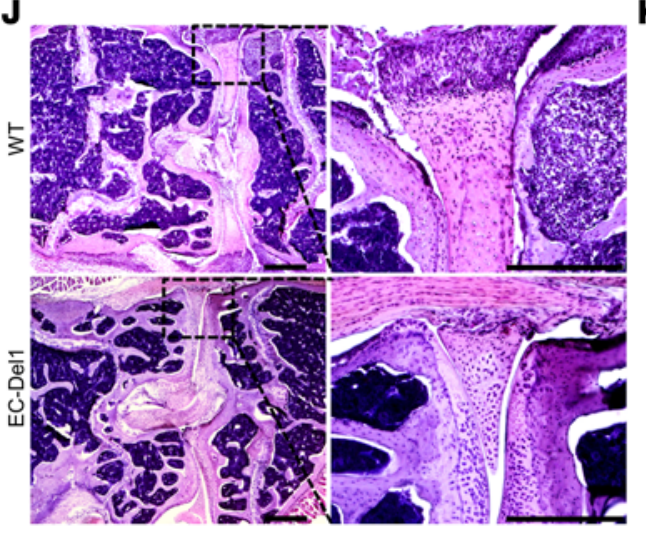

H

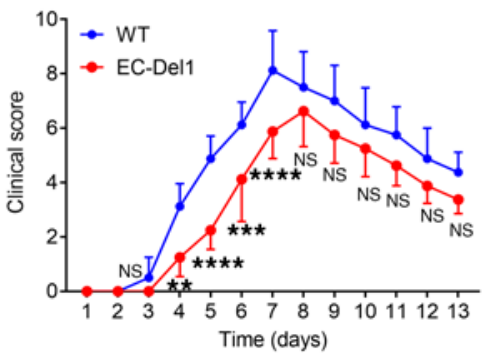

$\mathbf{K}$

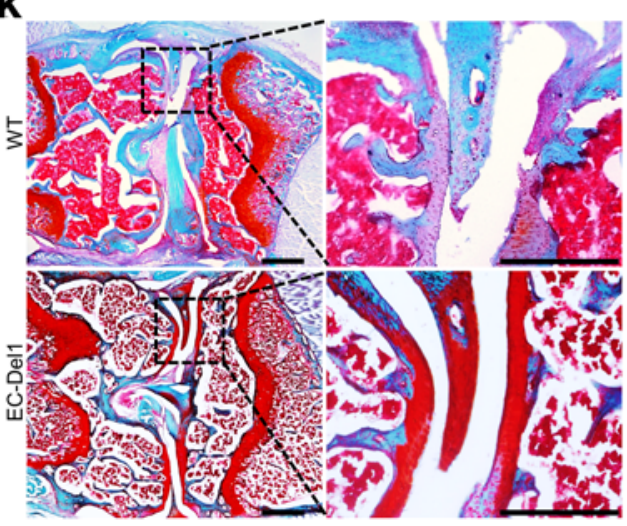

\section{I}

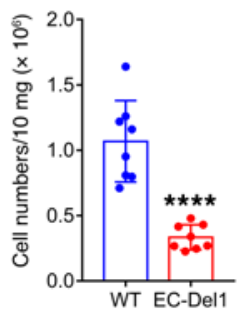

$\mathbf{L}$

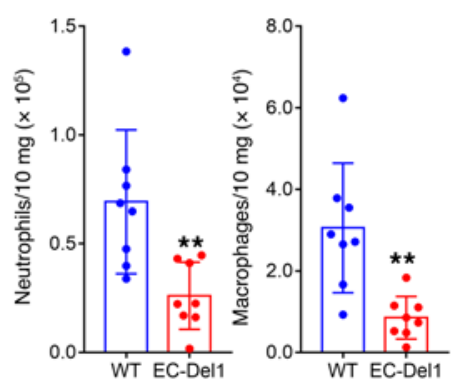

Figure 2. EC-Del1 mice are protected from CIA and CAIA relative to WT littermate controls. (A-F) CIA was induced by i.d. injection of $2 \mathrm{mg} / \mathrm{mL}$ CII emulsified with $2 \mathrm{mg} / \mathrm{mL}$ CFA into the tail of EC-Del1 mice and WT littermates. Joint thickness (A) and clinical scores (B) were recorded at indicated time points. (C) Total cell numbers in the synovium of knee joints in EC-Del1 and WT mice on day 42. H\&E (D) and safranin-O (E) staining of tissue sections from knee joints harvested on day 42. Scale bars: $500 \mu \mathrm{m}$. (F) Cell suspensions prepared from the synovium of knee joints (harvested on day 42) were processed for

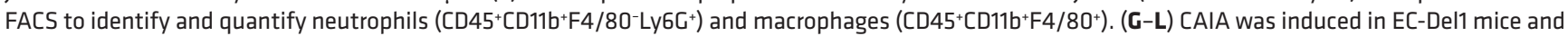
WT littermates by i.v. injection of arthritogenic mAbs $(0.5 \mathrm{mg} /$ mouse) at day 0 followed by i.p. injection of $50 \mu \mathrm{g}$ LPS on day 3 . Joint thickness (C) and clinical scores $(\mathbf{H})$ were recorded every day. (I) Total cell numbers in the synovium of knee joints in EC-Del1 and WT mice on day 7. H\&E (J) and safranin-O (K) staining of tissue sections from knee joints harvested on day 7. Scale bars: $500 \mu \mathrm{m}$. (L) Cell suspensions prepared from the synovium of knee joints (harvested on day

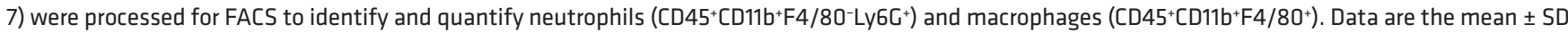
(A, B, C, F, G, H, I, and L: $n=8$ mice/group in total from 2 independent experiments). ${ }^{*} P<0.05 ;{ }^{* *} P<0.01 ;{ }^{* *} P<0.001 ;{ }^{* * *} P<0.0001$. Two-way ANOVA with repeated-measures and Sidak's post tests for comparison with WT mice (A, B, G, and $\mathbf{H})$ and Student's unpaired $t$ test (C, F, I, and $\mathbf{L})$. 
A

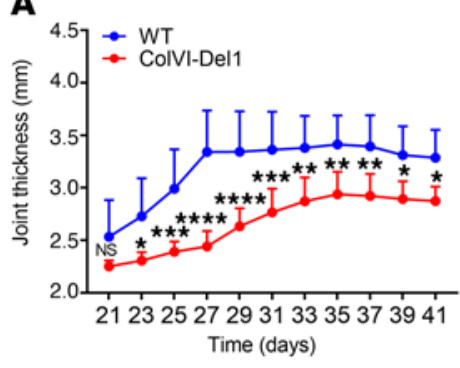

B

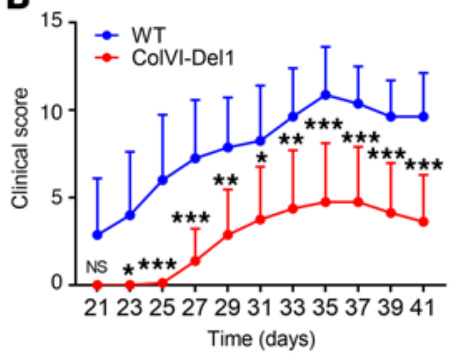

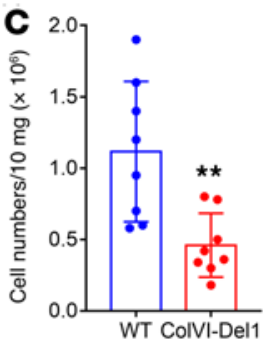

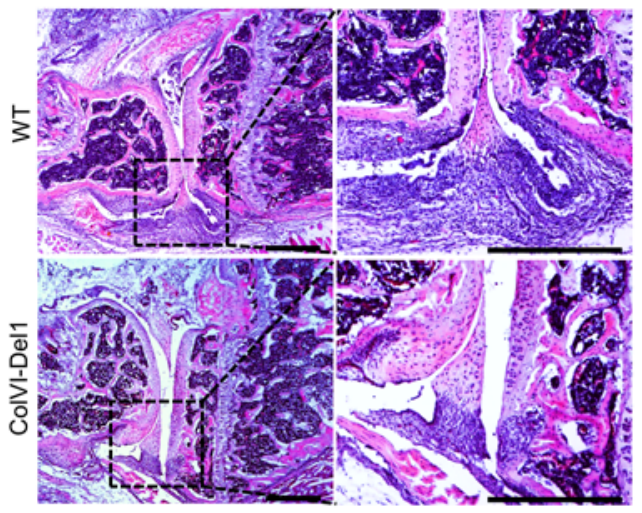

$\mathbf{F}$
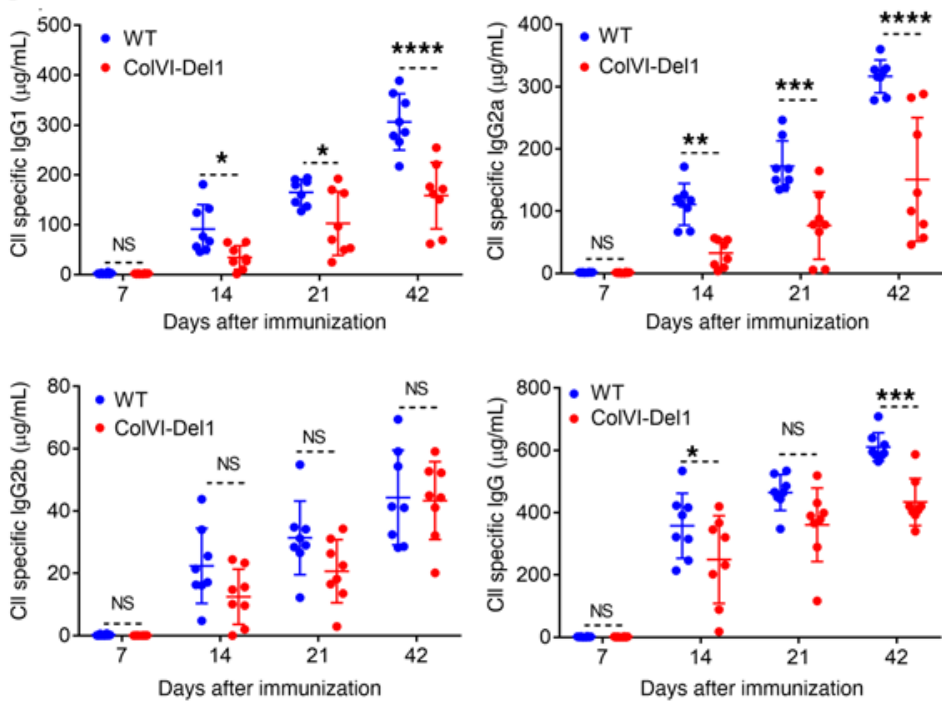

E

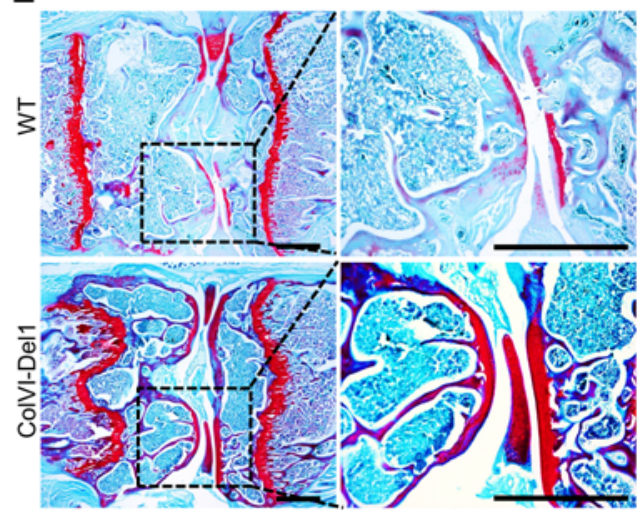

G

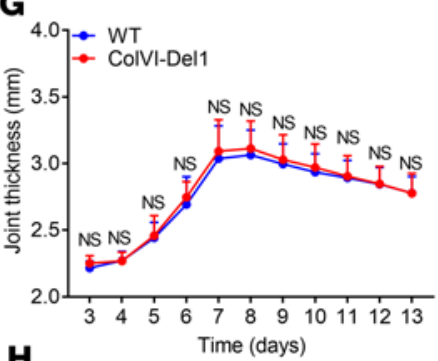

H

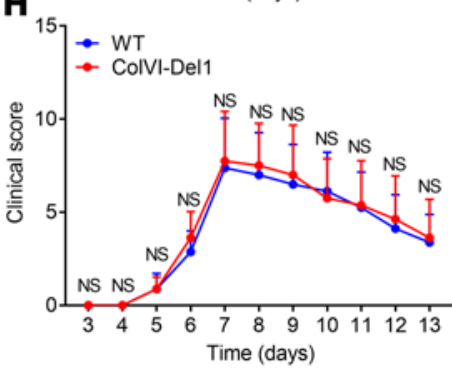

Figure 3. ColVI-Del1 mice are protected from CIA but not CAIA compared with WT littermate controls. (A-F) CIA was induced by i.d. injection of 2 mg/mL type II collagen (CII) emulsified with $2 \mathrm{mg} / \mathrm{mL}$ CFA into the tail of ColVI-Del1 mice and WT littermates. Joint thickness (A) and clinical score (B) were recorded at indicated time points. (C) Total cell numbers in the synovium of knee joints in ColVI-Del1 and WT mice on day 42 . H\&E (D) and safranin-O (E) staining of tissue sections from knee joints harvested on day 42 . Scale bars: $500 \mu \mathrm{m}$. (F) On days 7, 14, 21, and 42, sera were collected and isotype-specific and total IgC Abs to CII were measured by ELISA. (G and H) CAIA was induced in ColVI-Del1 mice and WT littermates by i.v. injection of arthritogenic mAbs ( 0.5 mg/ mouse) at day 0 followed by i.p. injection of $50 \mu \mathrm{g}$ LPS on day 3. Joint thickness $(\mathbf{C})$ and clinical scores $(\mathbf{H})$ were recorded every day. Data are the mean \pm SD ( $n=8$ mice/group, from 2 independent experiments). ${ }^{*} P<0.05 ;{ }^{* *} P<0.01 ;{ }^{* *} P<0.001 ;{ }^{* * *} P<0.0001$. Two-way ANOVA with repeated-measures and Sidak's post tests for comparison with WT mice (A, B, F, G, and $\mathbf{H})$ and Student's unpaired $t$ test (C).

DEL-1 on T cells. DEL-1 also binds $\beta 2$ integrins, such as LFA-1, which interactswithligandsinan RGD-independentmanner(15,17,20).Tfh cells express a highly active form of the LFA- 1 integrin which, in the context of TCR activation, promotes the expression of BCL6 that is critical for early DC-dependent Tfh differentiation (8). We therefore next addressed whether the ability of DEL-1-Fc to inhibit DCdependent Tfh generation (vs. Fc control; Figure 5, A and B) requires the presence of LFA-1. Using the above-described coculture system with LPS-preactivated BMDCs and either WT or LFA-1-deficient (Itgal $^{-/-}$; hereafter referred to as Lfa1-KO) $\mathrm{CD}^{+} \mathrm{T}$ cells, we found that DEL-1-Fc inhibited the generation of Tfh cells (defined as either $\mathrm{CD} 4^{+} \mathrm{CXCR} 5^{+} \mathrm{PD}-\mathrm{1}^{\mathrm{hi}}$ or $\left.\mathrm{CD} 4^{+} \mathrm{CXCR} 5^{+} \mathrm{ICOS}^{+}\right)$only in the presence of LFA-1 (Figure 5, C and D). Regarding the 2 Fc groups (gray color bars), the frequency of generated Tfh cells was significantly 
A

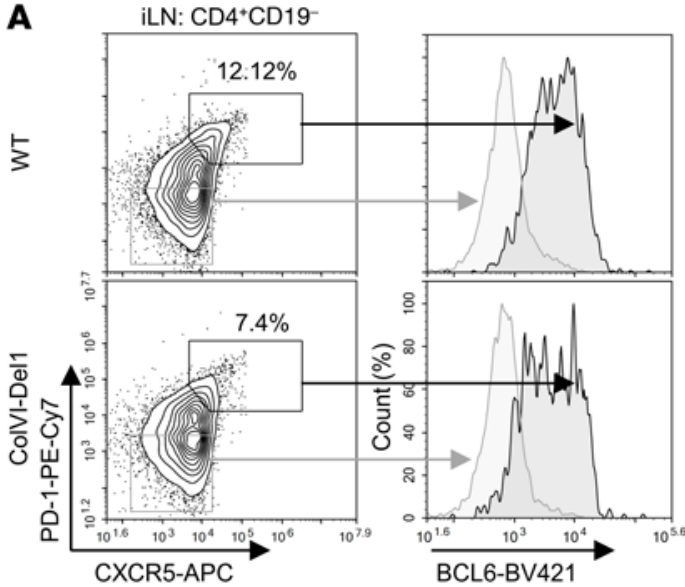

C

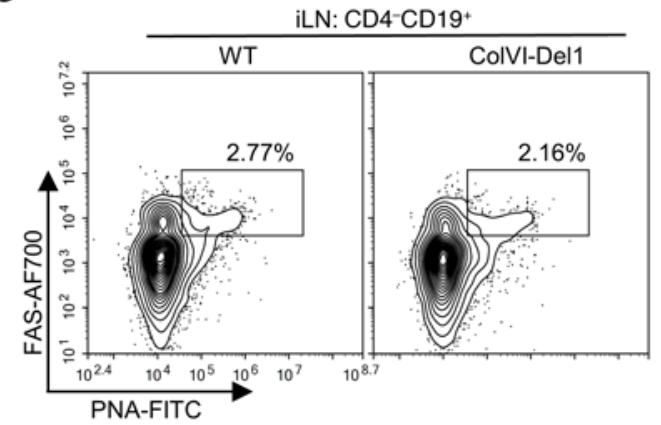

E

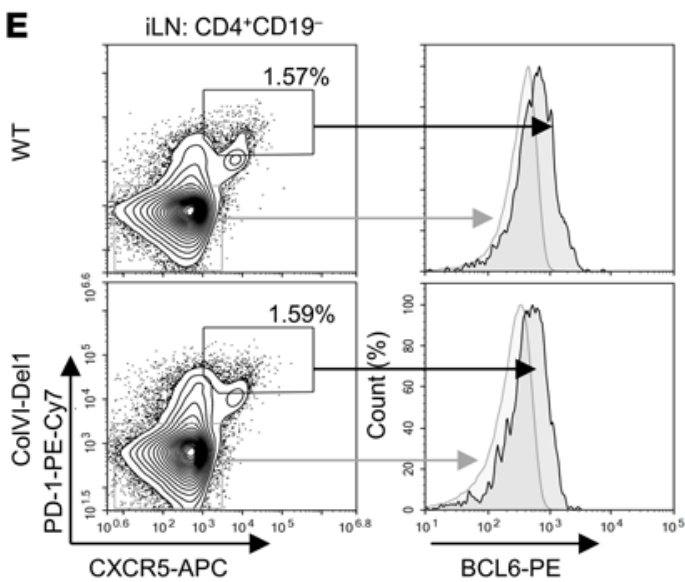

B
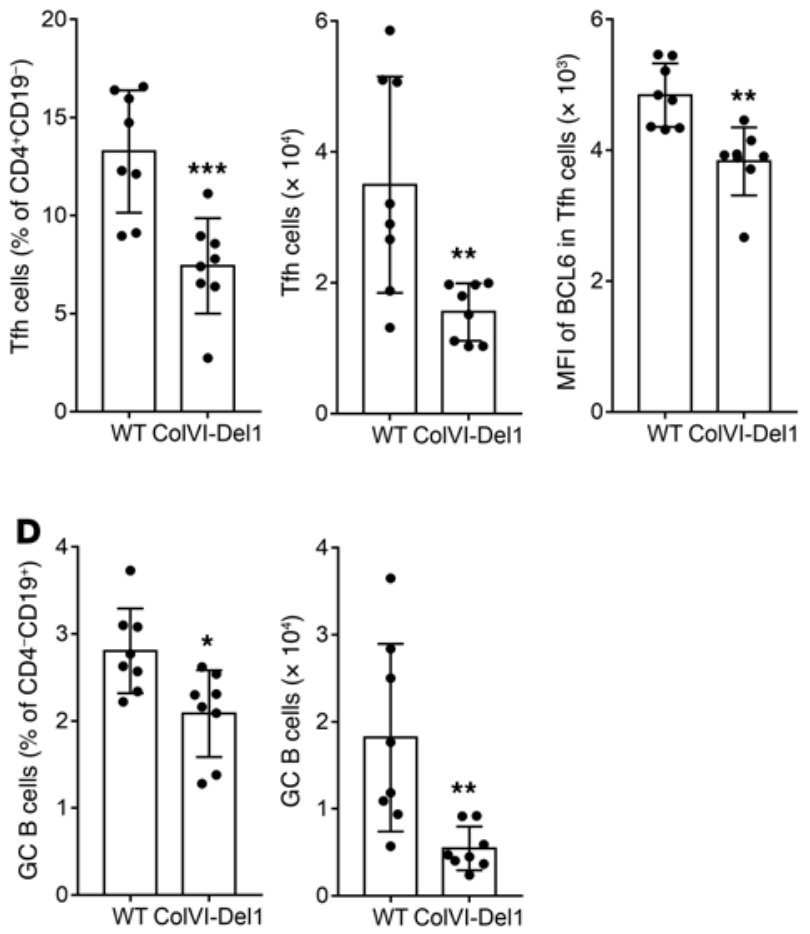

$\mathbf{F}$
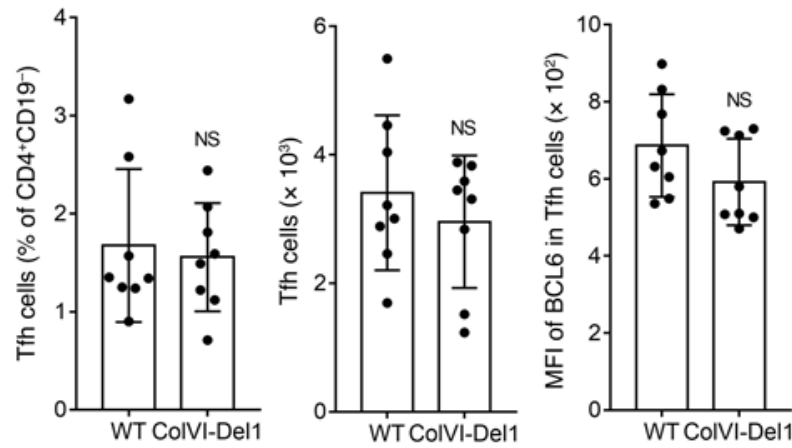

Figure 4. ColVI-Del1 mice have reduced numbers of Tfh and GC-B cells in inguinal LNs upon CIA but not at steady state. (A-D) CIA was induced by i.d. injection of $2 \mathrm{mg} / \mathrm{mL}$ Cll emulsified with $2 \mathrm{mg} / \mathrm{mL}$ CFA into the tail of ColVI-Del1 mice and WT littermates. On day 10, mononuclear cells from inguinal LNs were harvested and analyzed by FACS for the indicated Tfh and GC-B cell markers. (A) Representative FACS plots and (B) bar graphs of frequencies (left), absolute numbers (middle) and BCL6 MFI (right) of Tfh cells (CD4+CD19- CXCR5 + PD- $\left.1^{\text {hi }} B C L 6^{\text {hi }}\right)$. (C) Representative FACS plots and (D) bar graphs of frequencies (left) and absolute numbers (right) of GC-B cells (CD4- CD19+PNA+FAS+ cells). (E and F) Mononuclear cells of inguinal LNs from 7- to 8- week-old WT and ColVI-Del1 mice were harvested and analyzed by FACS for the indicated Tfh cell markers. (E) Representative FACS plots and (F) bar graphs of frequencies (left), absolute numbers (middle), and BCL6 MFI (right) of Tfh cells (CD4+CD19-CXCR5+PD-1 $\left.{ }^{\text {hi } B C L 6 ~} 6^{\text {hi}}\right)$. Data are the mean \pm SD (B, D, and F: $n=8$ mice/group from 2 independent experiments). ${ }^{*} P<0.05 ;{ }^{* *} P<0.01 ;{ }^{* *} P<0.001$. Student's unpaired $t$ test (B, left and middle, $\mathbf{D}$, and $\mathbf{F}$ ) and Mann-Whitney $U$ test (B, right).

decreased in the cocultures with Lfa1-KO CD4 ${ }^{+} \mathrm{T}$ cells relative to the cocultures with WT CD4 $4^{+}$T cells (Figure $5, \mathrm{C}$ and D), confirming the importance of LFA-1 in the induction of Thth differentiation (8).

DEL-1 inhibits BCL6 expression and activation of Tfh cells. Because BCL6 is critical for Tfh differentiation (3), we next determined whether the ability of DEL-1 to inhibit the induction of Tfh cells in the BMDC-T cell coculture system is associated with inhibition of
BCL6 expression. DEL-1-Fc caused a significant reduction in the expression of BCL6 in Tfh cells in the coculture system (Figure 5E), consistent with our in vivo finding that Tfh expression of BCL6 was decreased in the inguinal LNs of ColVI-Del1 mice relative to WT controls (Figure 4, A and B). Given that STAT3 is required for the ability of $\mathrm{CD} 4^{+} \mathrm{T}$ cells to efficiently express BCL6 and differentiate into Tfh cells $(37,38)$, we investigated whether DEL-1-Fc can also inhibit 
A
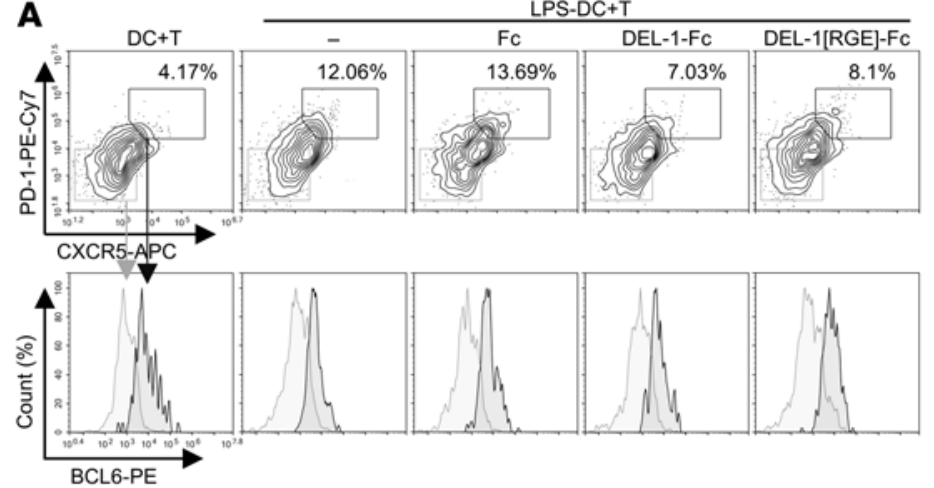

C $\quad L P S-D C+T(W T)$
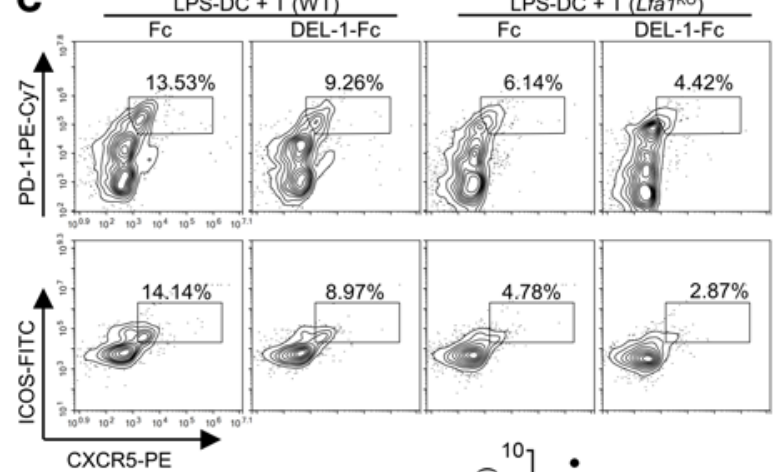

E
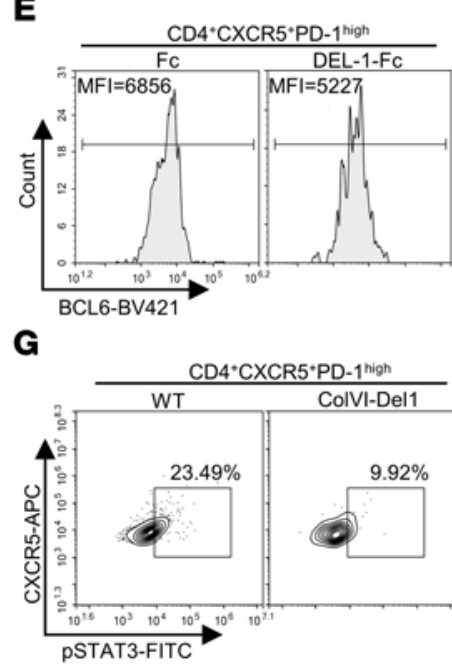

I
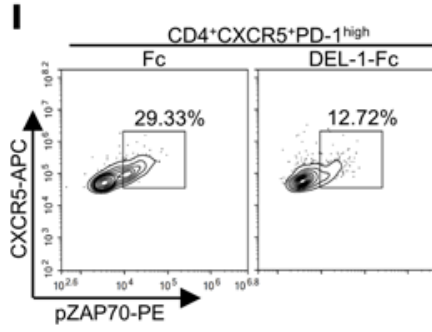

K

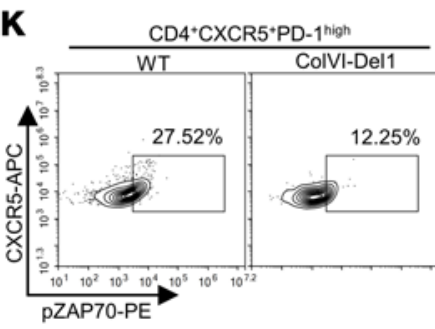

LPS-DC+T

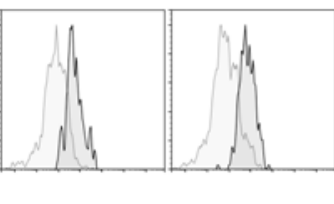

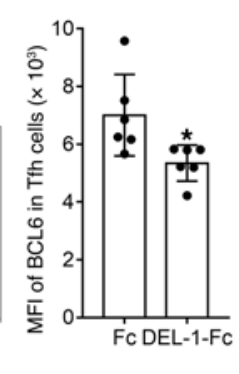

$\mathbf{F}$
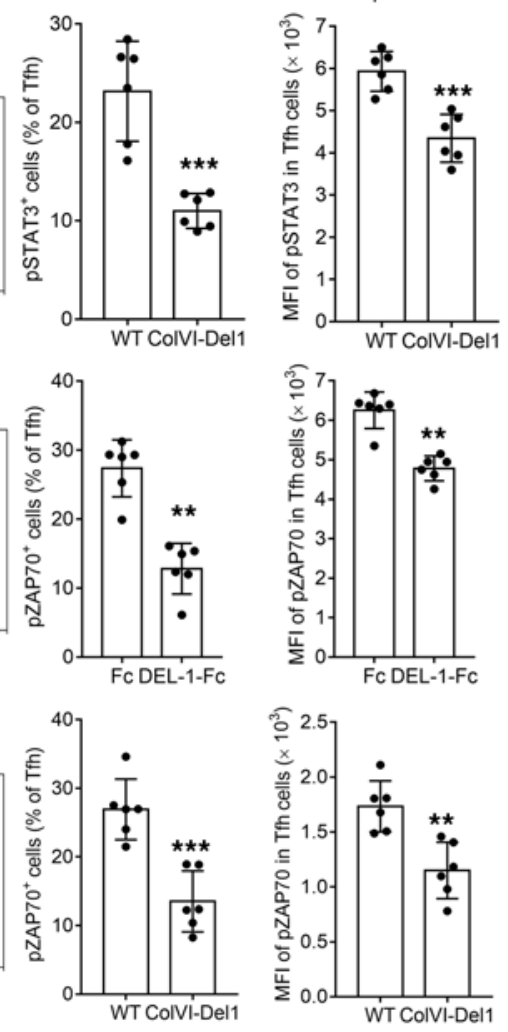

B

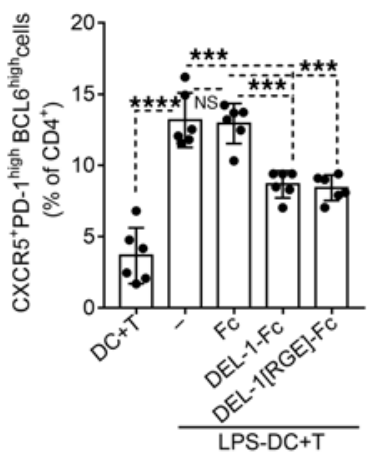

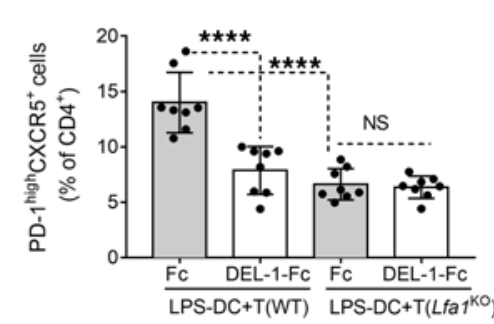
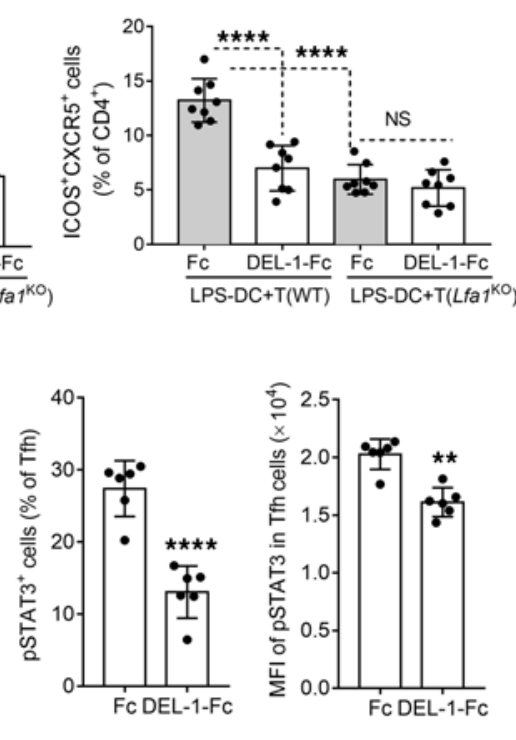

H
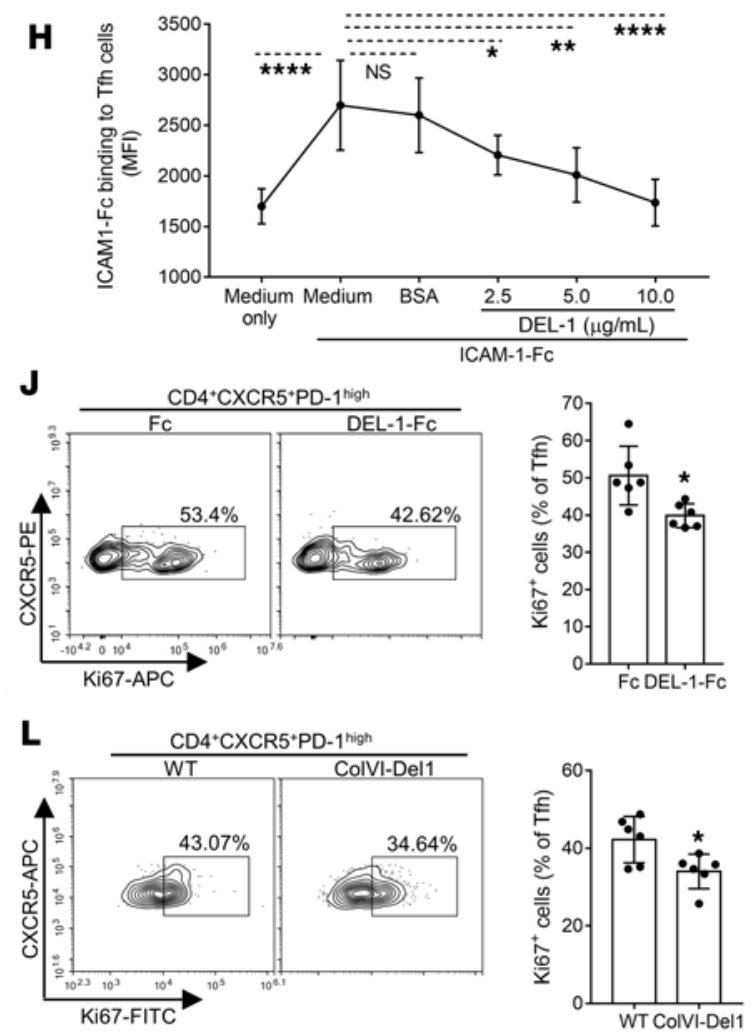
Figure 5. DEL-1 inhibits DC-dependent Tfh differentiation and activation. LPS-pretreated BMDCs were cocultured 7 days with anti-CD3/CD28-stimulated WT (A-F) or Lfa1-KO (C and D) CD4+ ${ }^{+}$cells, with or without DEL-1-Fc, DEL-1[RGE]-Fc, or Fc control (see Methods). Cells were analyzed by FACS: Representative plots (A and $\mathbf{C}$ ) and frequencies of Tfh cells, defined as CD4+CX-

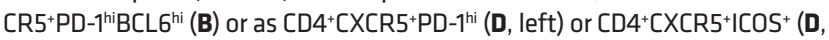
right). (E) FACS histograms and BCL6 MFI in CD4+CXCR5+PD- $1^{\text {hi }}$ T cells. (F) FACS plots (left) and frequencies of pSTAT3 ${ }^{+}$cells in CD4 $4^{+}$CXCR5 $5^{+} P D-1^{\text {hi }}$ T cells (middle) and pSTAT3 MFI (right). (C) CIA was induced in ColVI-Del1 mice and WT littermates and after 10 days inguinal LN mononuclear cells were analyzed: Representative plots (left) and frequencies of pSTAT3 ${ }^{+}$cells in Tfh cells (middle) and pSTAT3 MFI (right). (H) Binding of ICAM-1-Fc to Tfh cells (from LNs of mice subjected to $\mathrm{CI} A$ ) in the presence of DEL-1 or BSA control (see Methods). (I and J) Using the coculture system described above (A-F) with WT CD4+ T cells, the cells were analyzed after 3-day incubation: (I) FACS plots (left), frequencies of pZAP70+ cells (middle), and pZAP70 MFI (right) in CD4+ ${ }^{+} X-$ CR5 ${ }^{+} P D-1^{\text {hi }}$ T cells. (J) FACS plots (left) and frequencies of Ki67+ cells in CD4+CXCR5+PD-1hi T cells (right). ( $\mathbf{K}$ and $\mathbf{L}$ ) CIA was induced in ColVI-Del1 mice and WT littermates and after 10 days, inguinal LN mononuclear cells were analyzed: FACS plots (K and $\mathbf{L}$, left), frequencies of pZAP70+ (K, middle), or Ki67+ (L, right) cells and pZAP7O MFI (K, right) in Tfh (CD4 ${ }^{+}$CXCR5 $\left.{ }^{+} P D-1^{\text {hi) }}\right)$ cells. Data are the mean $\pm \mathrm{SD}$ (B, E, F, I, and $\mathbf{J} n=6$ cocultures/group; $\mathbf{D}, n=8$ cocultures; $\mathbf{G}, \mathbf{K}$, and $\mathbf{L} n=6$ mice/group, all from 2 independent experiments; $\mathbf{H}, n=6$ cultures/ group. ${ }^{*} P<0.05$; ${ }^{* *} P<0.01$; ${ }^{* *} P<0.001$; ${ }^{* * *} P<0.0001$. One-way ANOVA with Tukey's (B and $\mathbf{D}$ ) or Dunnett's post test (H); Student's unpaired $t$ test (E, $\mathbf{F}$ left, $\mathbf{G}, \mathbf{I}$ left, J, K, and $\mathbf{L}$ ); and Mann-Whitney $U$ test ( $\mathbf{F}$ and I right).

STAT3 activation. DEL-1-Fc caused a significant decrease in the frequency of pSTAT3-expressing Tfh cells and the pSTAT3 expression (MFI) level in the BMDC-T cell coculture system (Figure 5F). DEL-1 also inhibited STAT3 activation in vivo, as evidenced by reduced frequencies of $\mathrm{PSTAT}^{+}$cells in $\mathrm{CD} 4^{+} \mathrm{CXCR} 5^{+} \mathrm{PD}-\mathrm{1}^{\mathrm{hi}} \mathrm{Tfh}$ cells, as well as decreased pSTAT3 expression levels, at day 10 upon CIA in ColVIDel1 mice (Figure $5 \mathrm{G}$ ). Naive $\mathrm{CD} 4^{+} \mathrm{T}$ cells stimulated with anti-CD3 and ICAM-1 display enhanced BCL6 protein expression in comparison with anti-CD3 alone or with combined anti-CD3 and anti-CD28 stimulation (8). Therefore, the ability of DEL-1 to restrain the LFA-1dependent generation of Tfh cells suggests that DEL-1 might interfere with the interaction between ICAM- 1 on BMDCs and LFA- 1 on T cells. Consistently, DEL-1 dose-dependently inhibited the interaction of soluble ICAM- 1 with Tfh cells isolated from the inguinal LNs of mice subjected to CIA (Figure $5 \mathrm{H}$ ).

The protein tyrosine kinase ZAP-70 (zeta chain-associated protein of $70 \mathrm{kDa}$ ) plays a critical role in T cell activation (39) and can be activated by phosphorylation downstream of LFA-1 $(40,41)$. We thus examined whether DEL-1-Fc can inhibit the activation of ZAP-70 in the BMDC-T cell coculture system. DEL-1-Fc significantly reduced the frequency of $\mathrm{pZAP}^{+} \mathrm{O}^{+}$cells in the $\mathrm{CD} 4^{+} \mathrm{CXCR} 5^{+} \mathrm{PD}-1^{\mathrm{hi}} \mathrm{T}$ cell population (Figure 5I). Additionally, DEL-1-Fc inhibited BMDC-induced $\mathrm{CD}^{+} \mathrm{T}$ cell proliferation in the coculture system, as shown by significantly lower frequency of $\mathrm{Ki}-67^{+}$cells among the $\mathrm{CD} 4^{+} \mathrm{CX}-$ CR5 ${ }^{+} \mathrm{PD}-1^{\text {hi }} \mathrm{T}$ cell population than in cocultures treated with $\mathrm{Fc}$ control (Figure 5J). Tfh $\left(\mathrm{CD} 4^{+} \mathrm{CXCR} 5^{+} \mathrm{PD}-\mathrm{1}^{\mathrm{hi}}\right)$ cells from the inguinal LNs of ColVI-Del1 mice subjected to CIA contained lower frequencies of pZAP70 ${ }^{+}$cells (and reduced MFI levels of pZAP70; Figure $5 \mathrm{~K}$ ) and of $\mathrm{Ki}^{+}{ }^{+}$cells (Figure 5L), suggesting that DEL-1 overexpression inhibited the activation and proliferation of Tfh cells also in vivo. Taken together, the data from Figures 3-5 indicate that DEL-1 restrained arthritis, at least in part, by downregulating Tfh induction in LNs.
To determine whether the ability of DEL-1 to regulate Tfh cells in arthritis represents a general principle applying to different experimental settings, we used a model of parenteral sheep red blood cell (SRBC) immunization, which induces a Tfh cell-dependent GC response in LNs 7 to 9 days after challenge (42). Upon SRBC immunization, ColVI-Del1 mice displayed significantly reduced numbers of Tfh and GC-B cells than WT littermates on day 7 (Supplemental Figure 6, A and B). Consistently, serum anti-SRBC IgG levels were significantly lower in ColVI-Del1 mice than in their WT littermates on day 14 (Supplemental Figure 6C). At baseline, the 2 genotypes had similar serum concentrations of IgG isotypes (Supplemental Figure 6D); hence, ColVI-Del1 mice did not exhibit alterations at steady state. The total (antigen-nonspecific) concentrations of IgG1, IgG2a, and IgG2b were similar between ColVI-Del1 and WT controls at day 14 after immunization (Supplemental Figure 6D).

$L N$ stromal cell-derived DEL-1 inhibits Tfh and GC-B cell responses in an LFA-1-dependent manner in vivo. Since DEL-1 is a secreted protein that acts locally (14) and given our finding that DEL-1 regulates the numbers of Tfh cells in the inguinal LNs, we reasoned that DEL-1 is expressed in the inguinal LNs. Indeed, using an in situ RNA detection method, we localized DEL-1 mRNA expression in the T cell zone of the inguinal LNs and particularly near the T cell zone-B cell follicle interface ("T-B interface") in WT (but not Del1-KO) mice and ColVI-Del1 mice; the latter displayed increased DEL-1 mRNA expression relative to their WT littermates (Figure 6A). Because T cells do not express DEL-1 and stromal cells contribute to the regulation of the adaptive immune response in the LNs (by providing physical scaffold and critical factors for interactions between innate and adaptive immune cells; ref. 43), DEL-1 might be expressed by LN stromal cells. In this regard, Prados et al. showed that ColVI-Cre reporter mice (which share the same ColVI alpha1 promoter construct used in the generation of our ColVI-Del1 mice) target, among other mesenchymal cells in the Peyer's patches and intestinal lymphoid follicles, a population of perivascular stromal cells (pericytes) in all secondary lymphoid organs examined, including the LNs (44). Using RNA in situ hybridization and immunofluorescence microscopy, we showed that DEL-1 mRNA expression (detected in WT or ColVI-Del1 but not in Del1-KO LNs) colocalized with expression of CD146 and NG2 (Figure 6B), which are markers of pericytes (45). ELISA for DEL-1 of FACS-sorted CD146 ${ }^{+} \mathrm{NG}^{+}$pericytes (Supplemental Figure 1B) from LNs of ColVI-Del1 and WT mice demonstrated that pericytes produced DEL-1 and that its production was significantly higher in pericytes from ColVI-Del1 mice than from WT mice (Figure 6C). Expectedly, DEL-1 was not detected in the culture supernatants of CD $4^{+} \mathrm{T}$ cells (negative control) (Figure 6C). However, the finding that pericytes released DEL-1 did not necessarily indicate that the DEL-1-mediated inhibition of Tfh and GC-B cell responses in inguinal LNs of mice subjected to CIA (Figure 4) could be attributed to LN stromal cell-derived DEL-1.

To address this question, we performed LN transplantation experiments using a widely used model (46-49). Specifically, inguinal LNs of WT recipient mice were surgically replaced unilaterally by inguinal LNs from WT, ColVI-Del1, or Del1-KO mice (Figure 7A). In the transplanted LNs, the stromal cell compartment remains of donor origin, whereas the hematopoietic immune cells are essentially all host (recipient) derived $(48,49)$, which we confirmed using the CD45.1/CD45.2 congenic system (Supplemental Figure 7). After 4 
A

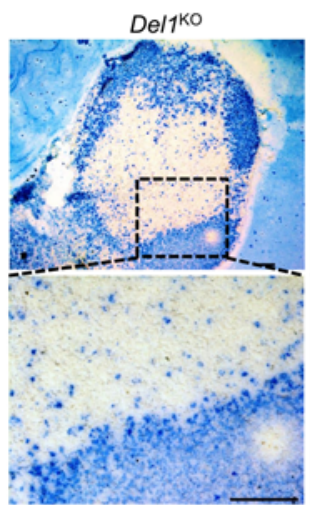

B
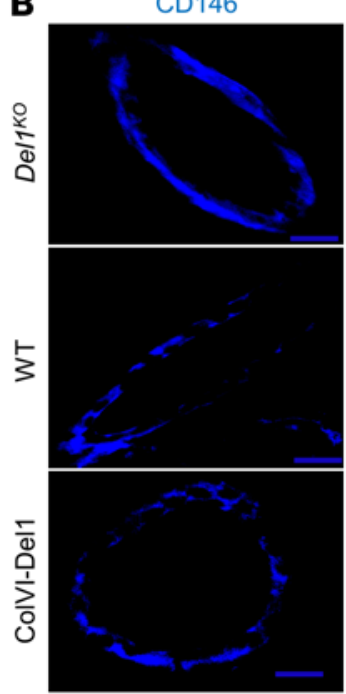

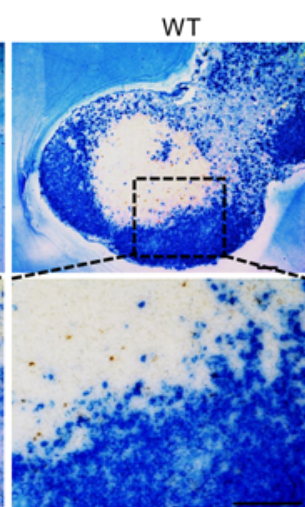

NG2
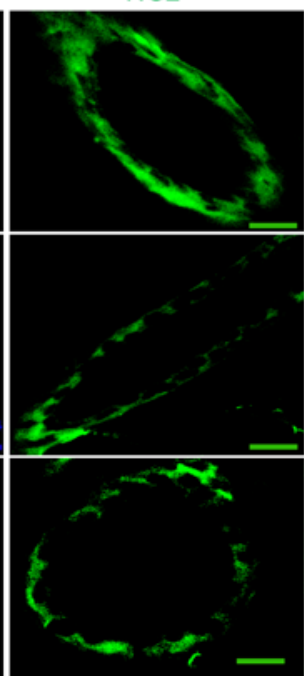

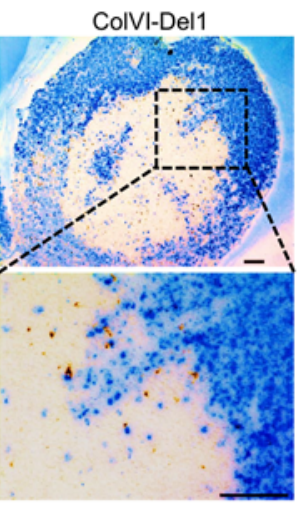

Del1

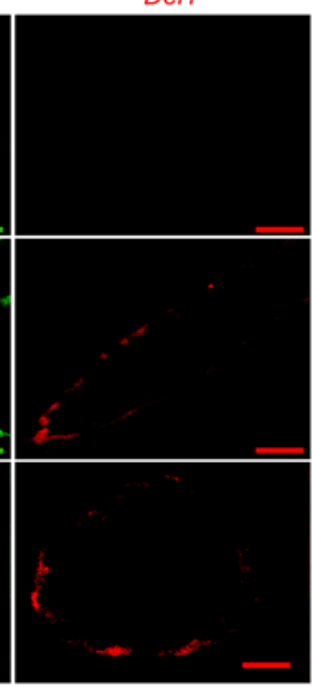

Merge

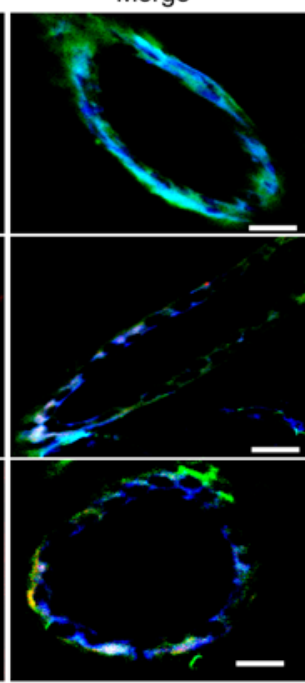

C

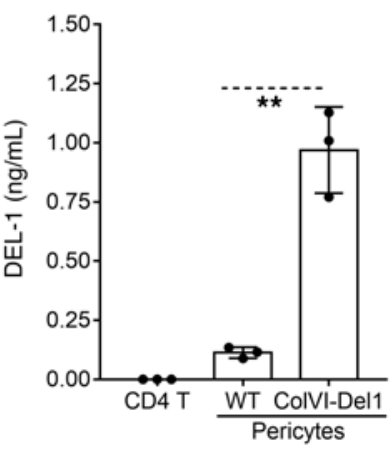

Figure 6. DEL-1 is expressed in the inguinal LNs by pericytes. (A) Inguinal LNs from 8- to 10-week-old Del1-KO, WT, and ColVI-Del1 mice at steady state were harvested to perform immunohistochemistry for B220 (pan-B cell marker; blue) and RNAscope in situ hybridization for DEL-1 mRNA expression (brown) (scale bars: $100 \mu \mathrm{m}$ ). (B) Immunofluorescence for the pericyte markers CD146 (blue) and NG2 (green) and RNAscope in situ hybridization for Del1 mRNA expression (red) (scale bars: $10 \mu \mathrm{m}$ ). (C) Inguinal LNs of ColVI-Del1 and WT mice were digested with collagenase IV and cell suspensions were stained with pericyte and T cell markers and sorted into CD45-CD31- $\mathrm{NC2}^{+} \mathrm{CD} 146^{+}$cells and CD4+ cells. The cells were cultured in vitro for 24 hours and the concentration of DEL- 1 in culture supernatants was measured by ELISA. Data are the mean \pm SD ( $n=3$ independent isolations/experiments). ${ }^{* *} P<0.01$; Student's unpaired $t$ test.

weeks, CIA was induced and 10 days later, mononuclear cells from the transplanted inguinal LNs were harvested and analyzed for Tfh and GC-B cell responses. Relative to the transplanted WT LN controls, the frequency and numbers of Tfh cells (defined as $\mathrm{CD}^{+} \mathrm{CD} 19^{-} \mathrm{CX}-$ CR5 ${ }^{+}$PD- $1^{\text {hi }}$ BCL6 ${ }^{\text {hi }}$ ) and their BCL6 expression level (MFI; Figure 7, B and $C$ ), as well as the frequency and numbers of $G C-B$ cells (defined as $\mathrm{CD}^{-} \mathrm{CD}^{-} 9^{+} \mathrm{PNA}^{+} \mathrm{FAS}^{+}$; Figure 7, D and E), were decreased significantly in transplanted LNs from ColVI-Del1 mice. Conversely, transplanted LNs from Del1-KO mice exhibited higher frequencies and numbers of Tfh (Figure 7, F and G) and GC-B cells (Figure 7, H and I) than those from WT controls. Further, independent evidence that LN-derived DEL-1 regulates Tfh and GC-B cell responses was obtained by showing that transplantation of WT LNs into Del1-KO hosts resulted in significant reduction of Tfh and GC-B cell responses in transplanted LNs upon induction of CIA compared with transplantation of Del1-KO LNs into Del1-KO hosts (Supplemental Figure 8).

Since DEL-1 inhibited DC-dependent Tfh differentiation in an LFA-1-dependent manner in vitro (Figure 5, C and D), we determined the in vivo physiological relevance of this observation using the LN transplantation system with Lfa1-KO mice serving as recipients (hence transplanted LNs will be repopulated by Lfa1KO hematopoietic cells). To this end, inguinal LNs of Lfa1-KO recipient mice were surgically replaced unilaterally by inguinal LNs from ColVI-Del1 (DEL-1 overexpression), Del1-KO (DEL-1 deficiency), or from Lfa1-KO donors (serving as control for the surgical treatment per se but also a condition of normal levels of DEL-1 expression). The recipient Lfa1-KO mice were subjected to CIA and downstream analysis as performed above with WT recipients. Analysis of the transplanted LNs revealed no DEL-1 expression-dependent differences in terms of the frequency and numbers of Tfh or GC-B cells (Supplemental Figure 9). In other words, in Lfa1-KO recipient mice, the Tfh and GC-B cell responses were similar regardless of the donor origin of the LNs, in contrast to the significant differences observed in WT recipients (Figure 7, B-I). Taken together, these data indicate that stromal cell-derived DEL1 inhibited Tfh and GC-B cells in vivo in a manner that required the presence of LFA- 1 in hematopoietic cells.

Tfh and GC-B cell responses in inguinal LNs upon CIA in EC-Del1 and Del1-KO mice relative to littermate controls. To further investigate the protective mechanism mediated by endothelial cell-derived 
A

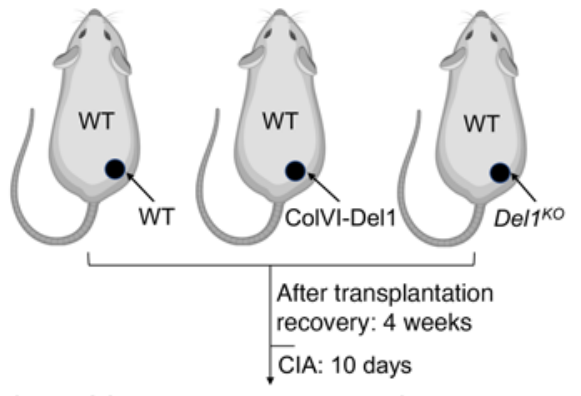

Tfh and GC B cell response analysis of transplanted LNs

\section{C}

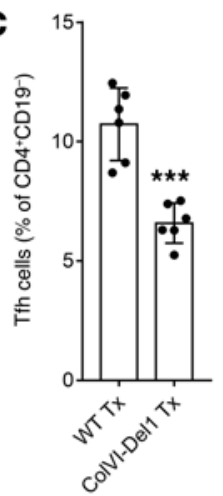

E
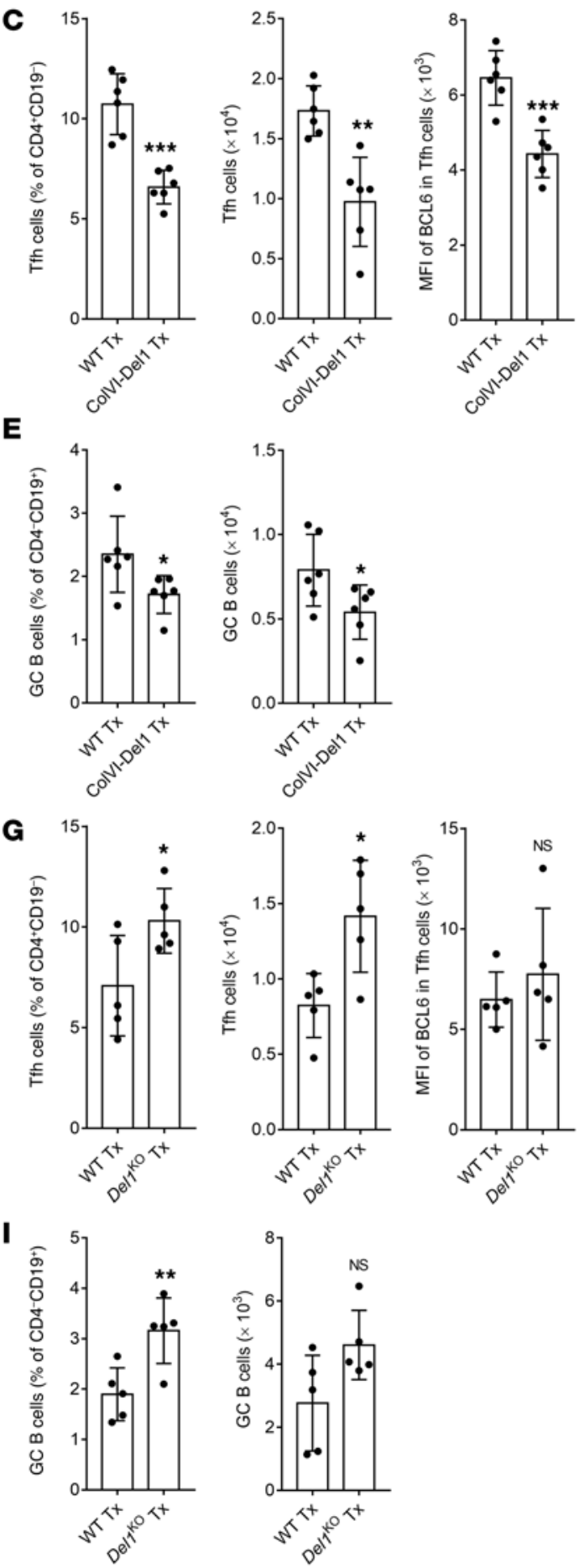

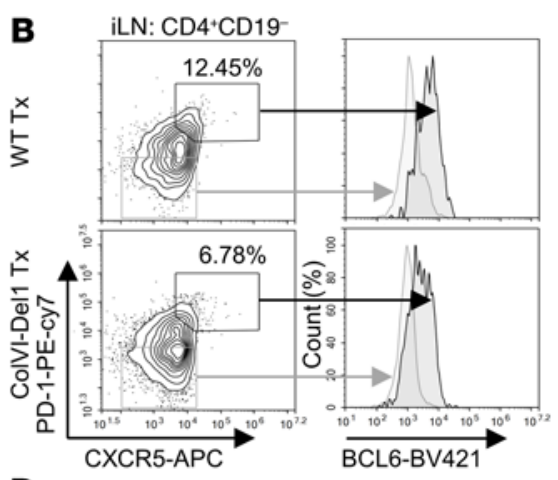

D
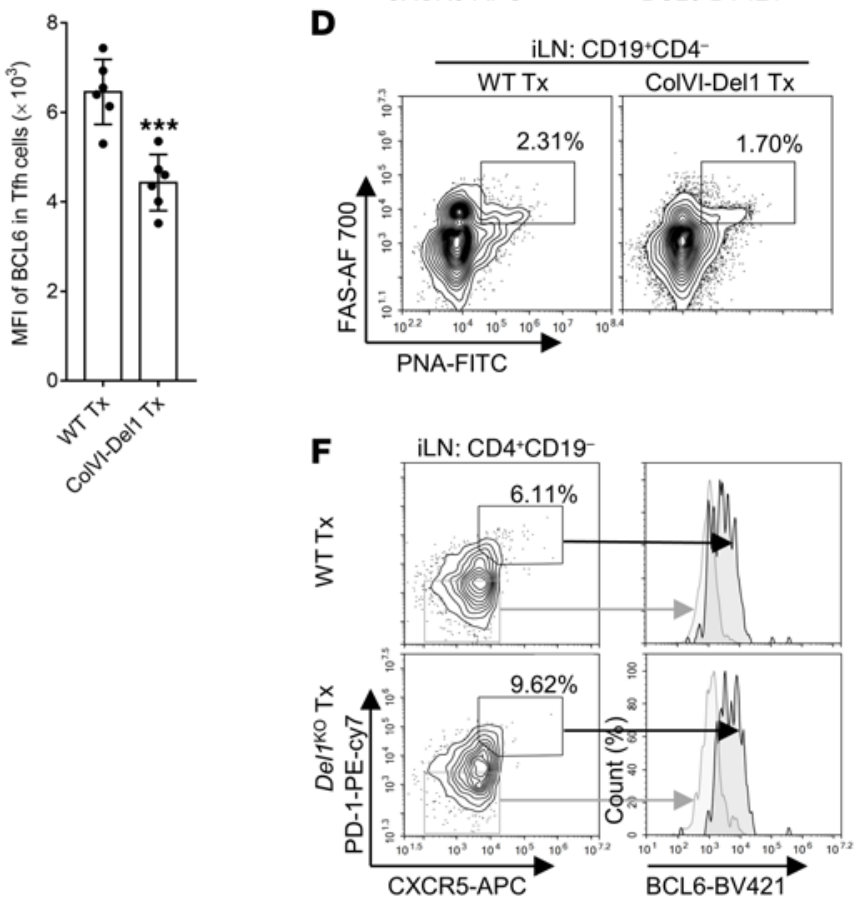

H

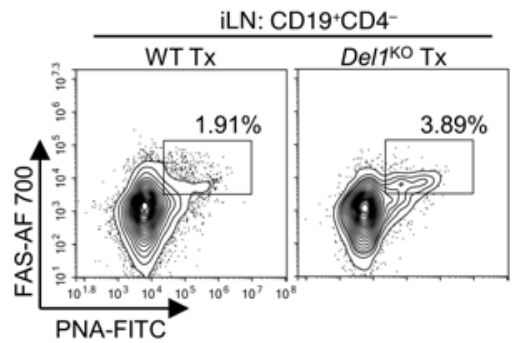

Figure 7. Lymph node stromal cell-derived DEL-1 inhibits Tfh and GC-B cell responses in vivo.

(A) LN transplantation-based experimental design to study the function of LN stromal cellderived DEL-1. Inguinal LNs of WT recipient mice were surgically replaced unilaterally by inguinal LNs from ColVI-Del1 (B-E) or Del1$\mathrm{KO}(\mathbf{F}-\mathbf{I})$ mice or their respective WT controls. (B-I) After 4 weeks, $\mathrm{CIA}$ was induced in the recipient mice by i.d. injection into the tail of $2 \mathrm{mg} / \mathrm{mL}$ Cll emulsified with $2 \mathrm{mg} / \mathrm{mL}$ (B-E) or $1 \mathrm{mg} / \mathrm{mL}$ (F-I) CFA. On day 10 , mononuclear cells from the transplanted inguinal LNs were harvested and analyzed for the indicated Tfh and GC-B cell markers by flow cytometry. (B and F) Representative FACS plots and (C and $\mathbf{G}$ ) frequencies and numbers of Tfh cells defined as CD4+CD19-CXCR5+PD- $1^{\text {hi }} B C L 6^{\text {hi }}$ cells (left and middle) and MFI of BCL6 expression (right) in $\mathrm{CD}^{+} \mathrm{CD}^{-} 9^{-}$ CXCR5+PD-1 ${ }^{\text {hi }}$ cells. (D and $\mathbf{H}$ ) Representative FACS plots and (E and I) frequencies and numbers of GC-B cells defined as CD4-CD19+ $P$ $\mathrm{NA}^{+} \mathrm{FAS}^{+}$cells. Data are the mean $\pm \mathrm{SD}$ ( $n=5-6$ mice/group). ${ }^{*} P<$ $0.05 ;{ }^{* *} P<0.01 ;{ }^{* *} P<0.001$. Student's unpaired $t$ test. 

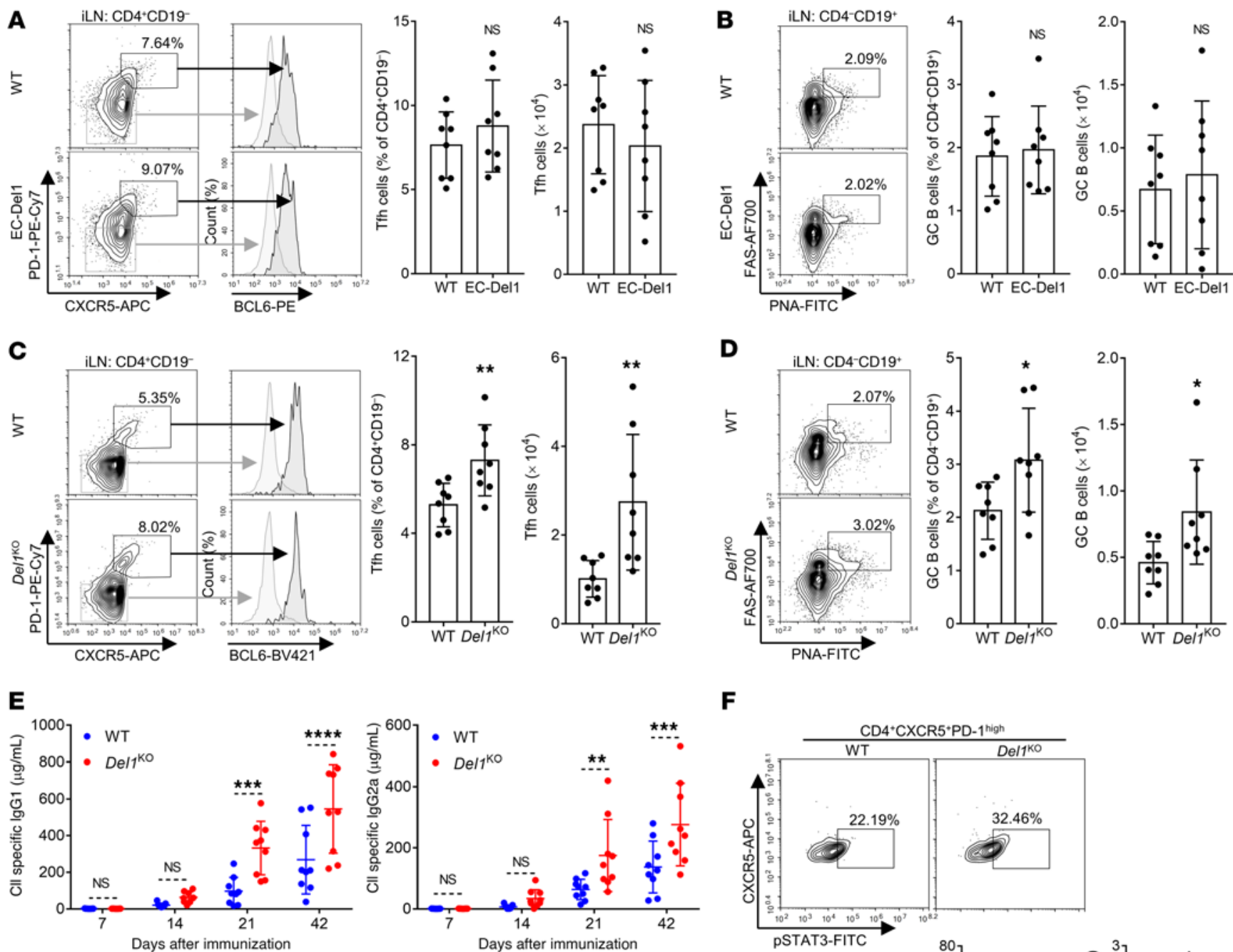

$\mathbf{F}$
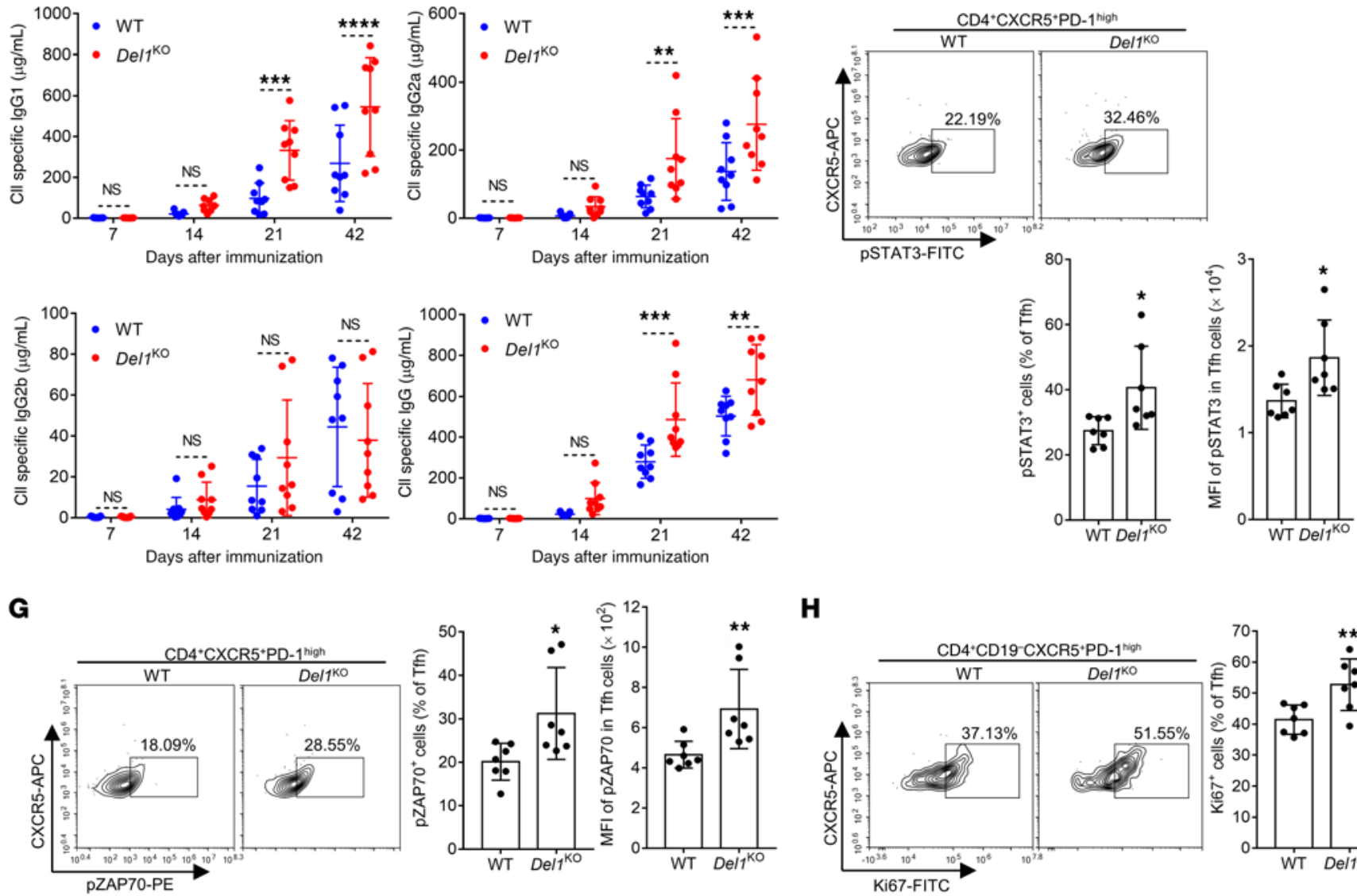

H

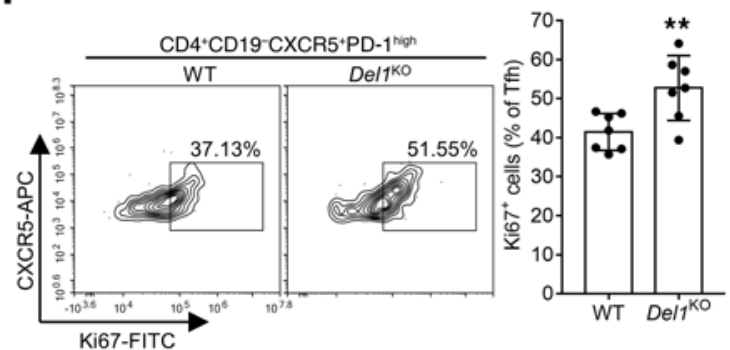


Figure 8. Tfh and GC-B cell responses in inguinal LNs upon CIA in EC-Del1 and Del1-KO mice. CIA was induced by i.d. injection (into the tail) of $2 \mathrm{mg} /$ $\mathrm{mL}$ Cll emulsified with $2 \mathrm{mg} / \mathrm{mL}$ CFA (in EC-Del1 mice and WT littermates; A and B) or with $1 \mathrm{mg} / \mathrm{mL}$ CFA (Del1-KO mice and WT littermates; C-H). After 10 days, mononuclear cells from inguinal LNs were harvested and analyzed for Tfh and GC-B cell markers by FACS. (A and C) Representative FACS plots (left) and frequencies and numbers of Tfh cells defined as CD4+CD19-CXCR5 ${ }^{+} P D-1^{\text {hi }} B-$ $\mathrm{CL}^{\text {hi }}$ cells (right) of indicated genotypes. (B and $\mathbf{D}$ ) Representative FACS plots (left) and frequencies and numbers of GC-B cells defined as CD4-CD19+PNA${ }^{+}$FAS' cells (right) of indicated genotypes. (E) On days 7, 14, 21, and 42, sera were collected from Del1-KO mice and WT littermates (same mice analyzed in Figure 1 for other parameters) and isotype-specific and total IgC Abs to CII were measured by ELISA. (F-H) 10 days after CIA induction, mononuclear cells from inguinal LNs of Del1-KO mice and WT littermates were harvested and analyzed by FACS for the indicated activation markers in Tfh cells. (F) Representative FACS plots (top) and frequencies of pSTAT3 ${ }^{+}$cells and MFI of pSTAT3 expression in Tfh (CD4+CXCR5+PD-1 hi) cells (bottom). (C) Representative FACS plots (left) and frequencies of pZAP70+ cells and MFI of pZAP70 expression in Tfh (CD4+CXCR5+PD-1 $\left.{ }^{\text {hi }}\right)$ cells (right). (H) Representative FACS plots (left) and frequencies of Ki-67+ cells in Tfh (CD4+CD19- CXCR5 $\left.{ }^{+} P D-1^{\text {hi }}\right)$ cells (right). Data are the mean $\pm \mathrm{SD}$ (A-D, $n=8$ mice/group; $\mathbf{E}, n=9$ mice/group; and $\mathbf{F}-\mathbf{H}, n$ $=7$ mice/group, all from 2 independent experiments). ${ }^{*} P<0.05 ;{ }^{*} P<0.01$; ${ }^{* * *} P<0.001$; ${ }^{* * *} P<0.0001$. Student's unpaired $t$ test (A-C, D left, $\mathbf{F}$, and $\left.\mathbf{H}\right)$ Mann-Whitney $U$ test (D right, $\mathbf{G}$ ); and 2-way ANOVA with repeated-measures and Sidak's post tests for comparison with WT mice (E).

DEL-1 in the CIA model, we compared EC-Del1 mice with their WT littermates in terms of Tfh and GC-B cell responses in the inguinal LNs. At day 10 upon CIA, EC-Del1 mice and WT littermate controls had similar frequencies and numbers of Tfh cells (defined as $\mathrm{CD}^{+}{ }^{+} \mathrm{CD} 19^{-} \mathrm{CXCR}^{+} \mathrm{PD}-1^{\text {hi }} \mathrm{BCL} 6^{\mathrm{hi}}$ ) and of GC-B cells (defined as $\mathrm{CD}^{-} \mathrm{CD}^{-}{ }^{+} \mathrm{PNA}^{+} \mathrm{FAS}^{+}$) in inguinal LNs (Figure 8, A and B). Thus, consistent with the concept that the location of DEL-1 expression dictates specific functions of this regulatory protein $(14,21)$, the overexpression of DEL-1 in endothelial cells did not affect Tfh/ GC-B cell responses, lending further support that the protection mediated by endothelial cell-derived DEL-1 is associated with its anti-recruitment function in the joints. In contrast, the exacerbation of CIA in DEL-1 deficiency (relative to their WT littermates) involved differential induction of Tfh and GC-B cell responses in the LNs. Specifically, in the absence of DEL-1 from the LNs, Del1$\mathrm{KO}$ mice exhibited higher frequencies and numbers of Tfh cells (Figure 8C) and GC-B cells (Figure 8D) in inguinal LNs than WT littermate controls. Consistent with these data, Del1-KO mice exhibited significantly higher levels of serum IgG1, IgG2a, and total IgG (but not IgG2b) CII-specific Abs than WT controls on days 21 and 42 after initiation of CIA (Figure 8E).

In contrast to our findings from the CIA model, at steady state, Del1-KO mice and WT controls exhibited comparable frequencies and numbers of Tfh cells and GC-B cells (Supplemental Figure 10 $A$ and $B$, respectively) in the inguinal LNs. These data suggest that endogenous DEL-1 may act to inhibit Tfh induction and GC-B cell responses preferentially upon immunological stress (CIA) rather than under steady-state conditions. Consistently, the mRNA expression of Edil3 (the gene encoding DEL-1) was increased in CD45- stromal cells from the LNs upon induction of CIA relative to its steady-state levels (Supplemental Figure 10C, left), presumably reflecting the proliferation of stromal cells, including pericytes, due to CIA. The CIA-induced upregulation of Edil3 in stromal cells was associated with upregulation of Itgal (the gene encoding the CD11a subunit of LFA-1) in CD4 ${ }^{+}$T cells from the LNs (Supplemental Figure 10C, right). These findings explain, at least in part, why the LFA1 integrin antagonist DEL-1 acts to inhibit Tfh induction preferentially upon immunological stress, such as CIA.

Although Th1 and Th17 cells are involved in the pathogenesis of arthritis (11), these T cell effector subsets were not differentially affected in Del1-KO mice subjected to CIA relative to WT controls (Supplemental Figure 11A), indicating that DEL-1 deficiency specifically affected Tfh cells in LNs. Moreover, we determined the frequencies of PD $-1^{+} \mathrm{CXCR}^{-}$cells from the FACS data of this (Figure $8, C$ and D) and an earlier (Figure 4, A-D) experiment and found no significant differences in the frequencies of $\mathrm{PD} 1^{+} \mathrm{CXCR} 5^{-}$activated non-Tfh cells between Del1-KO or ColVI-Del1 mice and their corresponding WT littermate controls (Supplemental Figure 11B). In contrast to the in vivo findings, in the in vitro DC-T cell coculture system (described in Figure 5), DEL-1-Fc inhibited the activation of non-Tfh cells as evidenced by reduced frequencies of IFN- $\gamma^{+}, \mathrm{IL}-17^{+}$, $\mathrm{TNF}^{-}, \mathrm{IL}^{-2} 2^{+}$, and $\mathrm{Ki} 7^{+}$cells in $\mathrm{CD} 4^{+} \mathrm{CXCR} 5{ }^{-} \mathrm{PD}-1^{+} \mathrm{T}$ cells (Supplemental Figure 12, A-E) and of Th1 (IFN- $\left.\gamma^{+}\right)$and Th17 (IL-17+) cells in $\mathrm{CD}^{+} \mathrm{T}$ cells (Supplemental Figure 12, F and G). Hence, in the LNs, DEL-1 predominantly localizes near the T-B interface, thereby inhibiting mainly Tfh cells rather than non-Tfh effector T cells. In an in vitro coculture system that lacks the aforementioned location-related limitations, DEL-1 can influence different $T$ cell subsets as long as LFA-1 is involved in their activation.

Further comparison between Del1-KO mice and WT controls in the CIA model indicated significant differences in Tfh cell activation. Specifically, consistent with the ability of DEL-1-Fc to cause a significant decrease in the frequencies of $\mathrm{pSTAT}^{+}$, $\mathrm{pZAP}^{+}$, and $\mathrm{Ki}-67^{+}$ cells in the $\mathrm{CD} 4^{+} \mathrm{CXCR} 5^{+} \mathrm{PD}-1^{\text {hi }} \mathrm{T}$ cell population of the BMDC-T cell coculture system (Figure $5 \mathrm{~F}$, I, and J, respectively), Tfh $\left(\mathrm{CD} 4^{+} \mathrm{CX}-\right.$ CR5 ${ }^{+} \mathrm{PD}-1^{\mathrm{hi}}$ ) cells from the inguinal LNs of Del1-KO mice contained higher frequencies of pSTAT3 $^{+}$cells (Figure 8F), pZAP70 ${ }^{+}$cells (Figure $8 \mathrm{G}$ ), and $\mathrm{Ki}^{+} 7^{+}$cells (Figure $8 \mathrm{H}$ ), suggesting that endogenous DEL-1 inhibits the activation and proliferation of Tfh cells in vivo.

DEL-1 was previously implicated in regulating chondrocyte apoptosis in the joints (50). Examination of the articular cartilage of all 4 genotypes used in this study revealed that WT, Del1-KO, EC-Del1, and ColVI-Del1 mice had comparable articular joint thickness at steady state (Supplemental Figure 13). Therefore, the hereby documented differences of the 4 genotypes in the CIA and CAIA models are unlikely to be related to the status of their knee cartilage at steady state.

Taken together, our data provide rigorous support that DEL-1 restrains arthritis by interfering with the induction of $\mathrm{Tfh}$ responses and the production of arthritogenic Abs, in addition to its ability to inhibit local leukocyte recruitment to the joints.

\section{Discussion}

We have generated mechanistic evidence that DEL-1 may restrain arthritis through dual spatially and functionally distinct mechanisms: a local mechanism in the joints associated with the previously described anti-leukocyte recruitment function of endothelial cell-derived DEL-1 and a systemic mechanism associated with the ability of stromal cell-derived DEL-1 to attenuate Tfh and GC-B cell responses in the LNs, as described here. Although the dissection of the mechanisms whereby DEL-1 restrains arthritis was facilitated by 
using cell-specific overexpression systems, the discovered mechanisms are physiologically relevant, as shown by comparing Del1-KO mice with their WT littermates. Indeed, endogenous DEL-1 attenuated joint inflammation and damage in CIA and CAIA models of arthritis by inhibiting inflammatory cell accumulation in the joints and $\mathrm{Tfh}$ induction as well as GC-B cell responses in the LNs.

In comparison with their respective WT littermates, EC-Del1 mice but not ColVI-Del1 mice were protected from CAIA, a model that bypasses the induction of the arthritogenic $A b$ response. EC-Del1 mice exhibited less severe arthritis upon CIA, although Tfh and GC-B cell responses were comparable between EC-Del1 mice and WT controls. In both models, the protection of EC-Del1 mice was associated with decreased numbers of infiltrated neutrophils and macrophages in the joints. Therefore, the protective mechanism in EC-Del1 operates locally and is likely mediated by the overexpression of DEL-1 in endothelial cells, where DEL-1 exerts anti-leukocyte recruitment function (21). On the other hand, in ColVI-Del1 mice, synovial fibroblast-derived DEL-1 failed to mediate local protection in the joints. This failure of ColVI-Del1 mice to be protected against CAIA, together with the protection of the same mice against CIA, prompted us to seek and discover a mechanism whereby DEL-1 can downregulate the induction of the adaptive immune response. Since arthritis could be restrained in mice with overexpression of DEL-1 from a ColVI alpha1 promoter as long as the induction of arthritogenic Abs was not bypassed (by passive administration of premade Abs), we reasoned that the underlying mechanism is associated with the induction of the arthritogenic $\mathrm{Ab}$ response. Indeed, our subsequent findings demonstrated that DEL-1, expressed by pericytes in the LN and localized in the T cell zone near the T-B interface, regulated $\mathrm{Ab}$ production in the LNs. The DEL-1-mediated inhibition of the anti-CII Ab responses in the CIA model was attributed to the ability of pericyte-derived DEL-1 to downregulate Tfh and GC-B cell responses in the LNs. DEL-1 can associate with the extracellular matrix in the proximity of DEL-1secreting cells, and thus its diffusion is likely limited (14). Consequently, the location of DEL-1 expression becomes critical in terms of dictating distinct homeostatic functions. Indeed, using different transgenic mice with cell type-specific overexpression of DEL-1, we have recently ascribed the anti-leukocyte recruitment action of DEL-1 to endothelial cell-derived DEL-1 and its efferocytic/pro-resolving function to macrophage-derived DEL-1 (21). This "location principle" for the action of DEL-1 (14) probably explains the differential susceptibility of EC-Del1 and ColVI-Del1 to CAIA; in other words, the location of DEL-1 overexpression in the joints (endothelial cells vs. synovial fibroblasts) is critical for its ability to restrain inflammatory cell recruitment and exacerbation of arthritis.

The multifunctionality of DEL-1 is greatly enabled by its interaction with distinct integrins. As alluded to above, the anti-leukocyte recruitment function of DEL-1 depends on its interaction with the LFA- 1 integrin $(15,16)$. By interacting with $\alpha v \beta 3$ integrin on different cell targets, DEL-1 regulates myelopoiesis (51), efferocytosis $(21,23)$, osteogenic differentiation of progenitors (22), and regulatory T cell stability and function (24), and by binding $\alpha_{v} \beta_{6}$ integrin, DEL-1 regulates integrin-mediated activation of TGF- $\beta$ (52). In this study, we showed that the interaction of DEL-1 with the LFA-1 integrin on T cells enabled DEL-1 to interfere with DC-dependent induction of Tfh cells in vitro. Importantly, Tfh cell priming by DCs predominantly occurs in the T-B interface of the LNs (53) where DEL-1 is expressed, as shown in the present study. Consistent with the in vitro mechanistic data, our LN transplantation experiments showed that stromal cell-derived DEL-1 inhibited Tfh cell and GC-B cell induction in vivo in a manner dependent on the expression of the LFA-1 integrin in hematopoietic cells.

Our findings are consistent with earlier reports showing that LFA- 1 is required for optimal APC-dependent T cell activation and induction of $\mathrm{Ab}$ responses in mice and humans $(54,55)$. LFA- 1 was also shown to be required for in vivo Tfh cell differentiation in a $\mathrm{CD}^{+} \mathrm{T}$ cell-intrinsic manner (8). The mechanisms driving Tfh cell differentiation and function are largely conserved between humans and mice (56). Uniquely among other effector T cells, Th cells from both mice and humans express high levels of LFA-1 (8). Moreover, DEL-1 regulation and functions are similar in mice and humans (14). These observations suggest that DEL-1 might also regulate the arthritogenic $\mathrm{Ab}$ response in human RA.

Although specific cytokines have been associated with induction of lineage-defining transcription factors and differentiation of distinct T cell effector subsets (Th1, Th2, Th17, Treg cells), there are no known specific cytokines that can selectively and sufficiently polarize naive $\mathrm{T}$ cells to the Tfh subset. On the other hand, cell-surface molecules associated with costimulation or intercellular adhesive interactions (CD28, ICOS, LFA-1) can initiate and sustain Tfh differentiation $(4,8,34)$. Based upon this principle, earlier studies utilized a human coculture system with preactivated (e.g., by LPS) DCs and naive $\mathrm{T}$ cells leading to $\mathrm{Tfh}$ differentiation $(35,36)$. By adopting this experimental approach to the mouse system, we not only dissected the LFA- 1 dependence of the mechanism by which DEL-1 downregulates DC-dependent differentiation of Tfh cells, but also elucidated downstream signaling mechanisms that were further validated by in vivo observations. In an in vitro (DC-T cell coculture system) and in vivo (CIA model) setting, DEL-1 inhibited STAT3 phosphorylation and BCL6 expression, as well as the activation and proliferation of Tfh cells, as evidenced by analysis of the ZAP-70 and Ki67 markers, respectively. These findings account for the ability of DEL-1 to restrain Tfh induction, and hence GC-B cell responses, resulting in a failure to generate anti-CII Abs. Moreover, these data are also consistent with the requirement of LFA- 1 for DEL-1-mediated inhibition of Tfh cell induction. This is because, in the context of TCR activation, LFA-1 activates ZAP-70 and STAT3 and promotes the expression of BCL6 that is critical for early DC-dependent Tfh cell differentiation $(8,41,57)$. STAT3-deficient $\mathrm{CD} 4^{+} \mathrm{T}$ cells have decreased capacity to differentiate into Tfh cells (37) and STAT3-deficient Tfh cells have decreased expression of BCL6 (38). The interaction of ICAM-1 with LFA-1 leads to the phosphorylation of the LFA-1-associated ZAP-70 kinase, which in turn contributes to shifting the conformation of LFA-1 from an intermediate- to a high-affinity state that potentiates its adhesive interactions with ICAM-1 (41). Interestingly, ZAP-70 defects have been associated with experimental arthritis in mice $(58,59)$. The ability of DEL-1 to block the ICAM-1-LFA-1 interaction should restrain both the aforementioned feed-forward mechanism that would amplify and sustain interactions between $\mathrm{T}$ cells (LFA-1) and APCs (ICAM-1), as well as downstream signaling events that promote BCL6-dependent Tfh differentiation. In this regard, persistent interactions with APCs are critical for maintaining BCL6 expression and driving Tfh differentiation (8). 
Since Th cells play a role in various autoimmune diseases, such as systemic lupus erythematosus (60), atopic dermatitis (61), multiple sclerosis (62), type 1 diabetes (63), and asthma (64), LN stromal cellderived DEL-1 may have a protective function in these diseases by downregulating the generation of Tfh cells. This activity of DEL-1 is not restricted to the context of autoimmunity since Tfh and GC-B cell responses were inhibited in ColVI-Del1 mice in the setting of SRBC immunization. Given that the induction of Tfh cells is crucial for Abmediatedprotectionagainstinfectionwithextracellularpathogens(56), future research may explore whether DEL-1 could be therapeutically manipulated in the LNs to promote protective humoral immunity.

In conclusion, DEL-1 restrains inflammatory arthritis by interfering with the induction of the arthritogenic $\mathrm{Ab}$ response in the LNs, where it is expressed by pericytes, and by limiting inflammatory cell recruitment to the joints by virtue of its expression by endothelial cells. These findings lend further support to the spatial regulation of the immune response (location principle) by DEL-1, which is critical for homeostatic immunity (14). EDIL3, the DEL-1-encoding gene, is a susceptibility locus in ankylosing spondylitis, a type of inflammatory arthritis that affects the vertebrae (65). Moreover, the DEL-1 protein is enriched in the synovial fluid of RA patients with low-level inflammation as compared with RA patients with high-level inflammation (66), suggesting that DEL-1 might play a protective role in human RA. These reports and our findings from the present study, therefore, suggest that DEL-1 may be a promising therapeutic for the treatment of inflammatory arthritis.

\section{Methods}

Mice. Mice were housed under specific pathogen-free conditions on a standard 12-hour light/12-hour dark cycle and were provided food and water ad libitum. Sex- and age-matched mice were used for experiments at 7-8 weeks of age. Because there were no significant differences in the results obtained with male and female mice, the respective data were pooled per treatment group. DBA/1OlaHsd mice, which are homozygous for the MHC class II allele H-2 (67), were obtained from Envigo. C57BL/6 Edil3-/- (Del1-KO) mice and C57BL/6 mice overexpressing DEL-1 in the endothelium (EC-Del1) were previously described (19, 27, 28). Mice deficient in the LFA-1 integrin (Itgal ${ }^{-/}$; designated Lfa1-KO in this study) and C57BL/6.SJL (CD45.1') mice were purchased from The Jackson Laboratory. Mice overexpressing DEL-1 from a ColVI alpha1 promoter (ColVI-Del1) were generated using the ColVI alpha1 promoter construct (30) (provided by George Kollias, BSRC Alexander Fleming, Vari, Greece), which was subcloned into the pBS KS vector backbone. Mouse Edil3 (encoding full-length DEL-1) was amplified by PCR and Edil3 cDNA was cloned into the pBS KS ColVI alpha1 vector and subsequently amplified in E. coli strain DH5. For generation of transgenic mice, the constructs were linearized and microinjected into pronuclei of oocytes from C57BL/6NCrl mice (Charles River Laboratory). Offspring were genotyped by PCR and transgene-bearing founders were further crossed with C57BL/6JOlaHsd mice (Envigo). After screening for transgene transmission to the offspring and Del1 overexpression by qPCR, 2 founders were selected, which were further backcrossed to the DBA/1 genetic background. Data are presented from one founder; key experiments were repeated using the second founder and similar results were obtained. To generate mice in the DBA/1 genetic background, ColVIDel1, EC-Del1, Del1-KO, and Lfa1-KO mice were mated with DBA/1 mice to generate F1 offspring. F1 male mice were then backcrossed sequentially to DBA/1 female mice until the F8 generation, and F8 mice were used for experiments.

CIA. CIA was induced by i.d. injection of immunization-grade chick CII (Chondrex) emulsified with CFA (Chondrex). Briefly, CFA at $2 \mathrm{mg}$ / $\mathrm{mL}$ (in studies using ColVI-Del1 or EC-Del1 mice together with respective WT littermates) or $1 \mathrm{mg} / \mathrm{mL}$ (in studies using Del1-KO mice and their littermate controls) and CII at $2 \mathrm{mg} / \mathrm{mL}$ were mixed thoroughly at a 1:1 ratio and emulsified using a syringe, obtaining the consistency of dense whipped cream. A $100 \mu \mathrm{L}$ volume of this emulsion was i.d. injected into the tail (68). From 21 days on, hind ankle joint thickness and clinical arthritis scores were recorded every other day according to a previously described scoring system on a 0 to 4 scale (68): 0 for normal (no erythema or swelling); 1 for erythema and mild swelling confined to the tarsals or ankle joint; 2 for erythema and mild swelling extending from the ankle to the tarsals; 3 for erythema and moderate swelling extending from the ankle to metatarsal joints; and 4 for erythema and severe swelling encompassing the ankle, foot and digits, or ankylosis of the limb. Clinical score was evaluated in a blinded manner for each hind paw and forepaw, and the final score for each mouse was the sum of the 4 paws. Hind ankle joint thickness was measured by using J 15 pocket dial thickness gauge (Käfer).

CAIA. CAIA was induced by i.v. injection of $0.5 \mathrm{mg}$ arthritogenic mAbs (Arthrogen-CIA 5-Clone Cocktail Kit; Chondrex) per mouse on day 0, followed by i.p. injection of $50 \mu \mathrm{g}$ LPS on day 3. Hind ankle joint thickness and clinical arthritis scores were recorded every day. The clinical scores were evaluated according to a previously described scoring system on a 0 to 4 scale (31): 0 for normal; 1 for mild redness, slight swelling of ankle or wrist; 2 for moderate swelling of ankle or wrist; 3 for severe swelling, including some digits, ankle and foot; 4 for maximally inflamed joint. Clinical score was evaluated in a blinded manner for each of hind paw and forepaw, and the final score for each mouse was the sum of the 4 paws.

LN transplantation. LN transplantation was performed as previously described $(46,47)$. Briefly, WT or Lfa1-KO recipient mice were anesthetized using ketamine/xylazine and s.c. injected with sustained-release buprenorphine and sustained-release meloxicam preoperatively. Inguinal LNs were removed by making an incision above the hind paw near the abdomen and replaced by inguinal LNs from donor mice into the fossa, and the skin was closed by stapling with Michel suture clips. After 4 weeks, mice were subjected or not to CIA, and LNs were collected for analysis.

For further technical information, see the Supplemental Methods.

Statistics. After confirming normality, data were evaluated by 1-way ANOVA followed by Tukey's or Dunnett's multiple-comparison test, as appropriate, or by 2-way repeated measures ANOVA and Sidak's multiple-comparison test. Where appropriate (comparison of 2 groups only), 2-tailed, unpaired Student's $t$ tests were performed. In cases in which the data did not follow normal distribution, nonparametric tests were performed, specifically a 2-tailed Mann-Whitney $U$ test (2-group comparisons) or Kruskal-Wallis test with Dunn's multiple-comparison test (comparisons involving more than 2 groups). All experiments were performed 2 or more times for verification. All statistical analyses were performed using GraphPad Prism software. P values less than 0.05 were considered to be statistically significant.

Study approval. Animal procedures were approved by the IACUC of the University of Pennsylvania.

\section{Author contributions}

HW designed and performed experiments, analyzed and interpreted data, and cowrote the manuscript. XL, TK, JHL, JS, SG, IK, and 
KN performed experiments and analyzed data. JMK analyzed and interpreted data. SG and RN generated new reagents. TC conceived and designed experiments, interpreted data, and edited the manuscript. GH conceived and designed the study, supervised research, interpreted data, cowrote the manuscript, and contributed to its editing. All authors read the manuscript and offered comments.

\section{Acknowledgments}

This work was supported by grants from the NIH (DE024153 and DE029436 to GH; DE028561 and DE026152 to GH and TC) and the German Research Foundation (SFB1181-project C07 to TC). We thank George Kollias (BSRC Alexander Fleming, Vari, Greece) for providing critical reagents and for critical assessment of the manuscript. The graphical abstract was made using BioRender.com.

Address correspondence to: George Hajishengallis, University of Pennsylvania, 240 S. 40th Street, Philadelphia, Pennsylvania 191046030, USA. Phone: 1.215.898.2091; Email: geoh@upenn.edu. JS’s present address is: Department of Nonclinical Study, CKD Research Institute, CKD Pharmaceutical Corporation, Seoul, South Korea.
1. Smolen JS, et al. Rheumatoid arthritis. Nat Rev Dis Primers. 2018;4:18001.

2. Qi H. T follicular helper cells in space-time. Nat Rev Immunol. 2016;16(10):612-625.

3. Hatzi K, et al. BCL6 orchestrates Tfh cell differentiation via multiple distinct mechanisms. J Exp Med. 2015;212(4):539-553.

4. Crotty S. Follicular helper CD4 T cells (TFH). Annu Rev Immunol. 2011;29:621-663.

5 . Biram A, et al. T cell interactions with B cells during germinal center formation, a three-step model. Immunol Rev. 2019;288(1):37-48.

6. Fazilleau N, et al. The function of follicular helper $\mathrm{T}$ cells is regulated by the strength of $\mathrm{T}$ cell antigen receptor binding. Nat Immunol. 2009;10(4):375-384.

7. Kandula S, Abraham C. LFA-1 on CD4+ T cells is required for optimal antigen-dependent activation in vivo. J Immunol. 2004;173(7):4443-4451.

8. Meli AP, et al. The integrin LFA-1 controls T follicular helper cell generation and maintenance. Immunity. 2016;45(4):831-846.

9. Nurieva RI, et al. Bcl6 mediates the development of T follicular helper cells. Science. 2009;325(5943):1001-1005.

10. Chang MH, Nigrovic PA. Antibody-dependent and -independent mechanisms of inflammatory arthritis. JCI Insight. 2019;4(5):e125278.

11. McInnes IB, Schett G. The pathogenesis of rheumatoid arthritis. N Engl JMed. 2011;365(23):2205-2219.

12. Cascao R, et al. Neutrophils in rheumatoid arthritis: more than simple final effectors. Autoimmun Rev. 2010;9(8):531-535.

13. Udalova IA, et al. Macrophage heterogeneity in the context of rheumatoid arthritis. Nat Rev Rheumatol. 2016;12(8):472-485.

14. Hajishengallis G, Chavakis T. DEL-1-regulated immune plasticity and inflammatory disorders. Trends Mol Med. 2019;25(5):444-459.

15. Choi EY, et al. Del-1, an endogenous leukocyte-endothelial adhesion inhibitor, limits inflammatory cell recruitment. Science. 2008;322(5904):1101-1104.

16. Eskan MA, et al. The leukocyte integrin antagonist Del-1 inhibits IL-17-mediated inflammatory bone loss. Nat Immunol. 2012;13(5):465-473.

17. Mitroulis I, et al. Developmental endothelial locus-1 attenuates complement-dependent phagocytosis through inhibition of Mac-1-integrin. Thromb Haemost. 2014;111(5):781-1006.

18. Choi EY, et al. Developmental endothelial locus-1 is a homeostatic factor in the central nervous system limiting neuroinflammation and demye- lination. Mol Psychiatry. 2015;20(7):880-888.

19. Kourtzelis I, et al. Developmental endothelial locus-1 modulates platelet-monocyte interactions and instant blood-mediated inflammatory reaction in islet transplantation. Thromb Haemost. 2016;115(4):781-788.

20. Shin J, et al. DEL-1 restrains osteoclastogenesis and inhibits inflammatory bone loss in nonhuman primates. Sci Transl Med. 2015;7(307):307ra155

21. Kourtzelis I, et al. DEL-1 promotes macrophage efferocytosis and clearance of inflammation. Nat Immunol. 2019;20(1):40-49.

22. Yuh DY, et al. The secreted protein DEL-1 activates a $\beta 3$ integrin-FAK-ERK1/2-RUNX2 pathway and promotes osteogenic differentiation and bone regeneration. J Biol Chem. 2020;295(21):7261-7273.

23. Hanayama R, et al. Expression of developmental endothelial locus- 1 in a subset of macrophages for engulfment of apoptotic cells. Jimmunol. 2004;172(6):3876-3882.

24. Li X, et al. The DEL-1/ $\beta 3$ integrin axis promotes regulatory $\mathrm{T}$ cell responses during inflammation resolution. J Clin Invest. 2020;130(12):6261-6277.

25. Kang YY, et al. Deficiency of developmental endothelial locus-1 (Del-1) aggravates bleomycin-induced pulmonary fibrosis in mice. Biochem Biophys Res Commun. 2014;445(2):369-374.

26. Yan S, et al. Developmental endothelial locus-1 (Del-1) antagonizes Interleukin-17-mediated allergic asthma. Immunol Cell Biol. 2018;96(5):526-535.

27. Chen LS, et al. Endothelial cell-specific overex pression of Del-1 drives expansion of haematopoietic progenitor cells in the bone marrow. Thromb Haemost. 2018;118(03):613-616.

28. Subramanian P, et al. Endothelial cell-specific overexpression of developmental endothelial locus-1 does not influence atherosclerosis development in ApoE $\mathrm{E}^{-/}$mice. Thromb Haemost. 2017;117(10):2003-2005.

29. Bartok B, Firestein GS. Fibroblast-like synoviocytes: key effector cells in rheumatoid arthritis. Immunol Rev. 2010;233(1):233-255.

30. Armaka M, et al. Mesenchymal cell targeting by TNF as a common pathogenic principle in chronic inflammatory joint and intestinal diseases. JExp Med. 2008;205(2):331-337.

31. Khachigian LM. Collagen antibody-induced arthritis. Nat Protoc. 2006;1(5):2512-2516.

32. Dahdah A, et al. Germinal center B cells are essential for collagen-induced arthritis. Arthritis Rheumatol. 2018;70(2):193-203.
33. Johnston RJ, et al. Bcl6 and Blimp-1 are reciprocal and antagonistic regulators of $\mathrm{T}$ follicular helper cell differentiation. Science. 2009;325(5943):1006-1010.

34. Choi YS, et al. ICOS receptor instructs $\mathrm{T}$ follicular helper cell versus effector cell differentiation via induction of the transcriptional repressor $\mathrm{Bcl} 6$. Immunity. 2011;34(6):932-946.

35 . Schmitt N, et al. Human dendritic cells induce the differentiation of interleukin-21-producing $\mathrm{T}$ follicular helper-like cells through interleukin-12. Immunity. 2009;31(1):158-169.

36. Pattarini L, et al. TSLP-activated dendritic cells induce human $\mathrm{T}$ follicular helper cell differentiation through OX40-ligand. JExp Med. 2017;214(5):1529-1546.

37. Nurieva RI, et al. Generation of T follicular helper cells is mediated by interleukin-21 but independent of T helper 1, 2, or 17 cell lineages. Immunity. 2008;29(1):138-149.

38. Ray John P, et al. Transcription factor STAT3 and type I interferons are corepressive insulators for differentiation of follicular helper and T helper 1 cells. Immunity. 2014;40(3):367-377.

39. Wang $\mathrm{H}$, et al. ZAP-70: an essential kinase in T-cell signaling. Cold Spring Harb Perspect Biol. 2010;2(5):a002279.

40. Verma NK, Kelleher D. Not just an adhesion molecule: LFA-1 contact tunes the T lymphocyte program. JImmunol. 2017;199(4):1213-1221.

41. Evans R, et al. The integrin LFA-1 signals through ZAP-70 to regulate expression of high-affinity LFA-1 on T lymphocytes. Blood. 2011;117(12):3331-3342.

42. Kim DH, et al. Estrogen receptor $\alpha$ in T cells suppresses follicular helper $\mathrm{T}$ cell responses and prevents autoimmunity. Exp Mol Med. 2019;51(4):1-9.

43. Krishnamurty AT, Turley SJ. Lymph node stromal cells: cartographers of the immune system. Nat Immunol. 2020;21(4):369-380.

44. Prados A, et al. CollagenVI-Cre mice: a new tool to target stromal cells in secondary lymphoid organs. Sci Rep. 2016;6:33027.

45. Robin C, et al. Human placenta is a potent hematopoietic niche containing hematopoietic stem and progenitor cells throughout development. Cell Stem Cell. 2009;5(4):385-395.

46. Baptista AP, et al. Lymph node stromal cells constrain immunity via MHC class II self-antigen presentation. Elife. 2014;3:e04433.

47. Dasoveanu DC, et al. Lymph node stromal CCL2 limits antibody responses. Sci Immunol. 2020;5(45):eaaw0693.

48. Ahrendt M, et al. Stromal cells confer lymph 
node-specific properties by shaping a unique microenvironment influencing local immune responses. J Immunol. 2008;181(3):1898-1907.

49. Hammerschmidt SI, et al. Stromal mesenteric lymph node cells are essential for the generation of gut-homing T cells in vivo. J Exp Med. 2008;205(11):2483-2490.

50. Wang Z, et al. Del1 knockout mice developed more severe osteoarthritis associated with increased susceptibility of chondrocytes to apoptosis. PLoS One. 2016;11(8):e0160684.

51. Mitroulis I, et al. Secreted protein Del-1 regulates myelopoiesis in the hematopoietic stem cell niche. J Clin Invest. 2017;127(10):3624-3639.

52. Kim DY, et al. Del-1, an endogenous inhibitor of TGF- $\beta$ activation, attenuates fibrosis. Front Immunol. 2020;11:68.

53. Krishnaswamy JK, et al. Determination of $\mathrm{T}$ follicular helper cell fate by dendritic cells. Front Immunol. 2018;9:2169.

54. Morrison VL, et al. Optimal T cell activation and $\mathrm{B}$ cell antibody responses in vivo require the interaction between leukocyte functionassociated antigen-1 and kindlin-3. J Immunol.
2015;195(1):105-115.

55. Fischer A, et al. Role of the LFA-1 molecule in cellular interactions required for antibody production in humans. JImmunol. 1986;136(9):3198-3203.

56. Tangye SG, et al. The good, the bad and the ugly - TFH cells in human health and disease. Nat Rev Immunol. 2013;13(6):412-426.

57. Verma NK, Kelleher D. Adaptor regulation of LFA-1 signaling in T lymphocyte migration: Potential druggable targets for immunotherapies? Eur J Immunol. 2014;44(12):3484-3499.

58. Kugyelka R, et al. ZAP-70 regulates autoimmune arthritis via alterations in $\mathrm{T}$ cell activation and apoptosis. Cells. 2019;8(5):504.

59. Au-Yeung BB, et al. ZAP-70 in signaling, biology, and disease. Annu Rev Immunol. 2018;36(1):127-156.

60. Faliti CE, et al. P2X7 receptor restrains pathogenic Tfh cell generation in systemic lupus erythematosus. J Exp Med. 2019;216(2):317-336.

61. Szabo K, et al. Expansion of circulating follicular $\mathrm{T}$ helper cells associates with disease severity in childhood atopic dermatitis. Immunol Lett. 2017;189:101-108.

62. Guo J, et al. T follicular helper-like cells are involved in the pathogenesis of experimental autoimmune encephalomyelitis. Front Immunol. 2018;9:944.

63. Kenefeck R, et al. Follicular helper $\mathrm{T}$ cell signature in type 1 diabetes. JClin Invest. 2015;125(1):292-303.

64. Ballesteros-Tato A, et al. T follicular helper cell plasticity shapes pathogenic $\mathrm{T}$ helper 2 cellmediated immunity to inhaled house dust mite. Immunity. 2016;44(2):259-273.

65. Lin Z, et al. A genome-wide association study in Han Chinese identifies new susceptibility loci for ankylosing spondylitis. Nat Genet. 2012;44(1):73-77.

66. Foers AD, et al. Proteomic analysis of extracellular vesicles reveals an immunogenic cargo in rheumatoid arthritis synovial fluid. Clin Transl Immunology. 2020;9(11):e1185.

67. Adarichev VA, et al. Major histocompatibility complex controls susceptibility and dominant inheritance, but not the severity of the disease in mouse models of rheumatoid arthritis. Immunogenetics. 2002;54(3):184-192.

68. Brand DD, et al. Collagen-induced arthritis. Nat Protoc. 2007;2(5):1269-1275. 